\title{
Quantum Gravity: Unification of Principles and Interactions, and Promises of Spectral Geometry
}

\author{
Bernhelm BOOSS-BAVNBEK ${ }^{\dagger}$, Giampiero ESPOSITO ${ }^{\ddagger}$ and Matthias LESCH ${ }^{\S}$ \\ † IMFUFA, Roskilde University, P.O. Box 260, 4000 Roskilde, Denmark \\ E-mail:booss@ruc.dk \\ URL: http://imfufa.ruc.dk/ Booss \\ $\ddagger$ INFN, Sezione di Napoli and Dipartimento di Scienze Fisiche, \\ Complesso Universitario di Monte S. Angelo, Via Cintia, Edificio 6, 80126 Napoli, Italy \\ E-mail: giampiero.esposito@na.infn.it \\ URL: http://www.dsf .unina.it/Theor/gruppoiv/gesposit.htm \\ $\S$ Bonn University, Mathematical Institute, Beringstr. 6, D-53115 Bonn, Germany \\ E-mail: lesch@math.uni-bonn.de \\ URL: http://www.math.uni-bonn.de/people/lesch
}

Received August 14, 2007, in final form September 25, 2007; Published online October 05, 2007

Original article is available at http://www.emis.de/journals/SIGMA/2007/098/

\begin{abstract}
Quantum gravity was born as that branch of modern theoretical physics that tries to unify its guiding principles, i.e., quantum mechanics and general relativity. Nowadays it is providing new insight into the unification of all fundamental interactions, while giving rise to new developments in modern mathematics. It is however unclear whether it will ever become a falsifiable physical theory, since it deals with Planck-scale physics. Reviewing a wide range of spectral geometry from index theory to spectral triples, we hope to dismiss the general opinion that the mere mathematical complexity of the unification programme will obstruct that programme.
\end{abstract}

Key words: general relativity; quantum mechanics; quantum gravity; spectral geometry

2000 Mathematics Subject Classification: 83C45; 83E05; 83E30; 83E50; 58B34; 58J20; $58 \mathrm{~J} 28 ; 58 \mathrm{~J} 30 ; 58 \mathrm{~J} 32 ; 58 \mathrm{~J} 52$

In memory of Tom Branson

\section{Introduction}

\section{Lorentzian spacetime and gravity}

In modern physics, thanks to the work of Einstein [39], space and time are unified into the spacetime manifold $(M, g)$, where the metric $g$ is a real-valued symmetric bilinear map

$$
g: T_{p}(M) \times T_{p}(M) \rightarrow \mathbb{R}
$$

of Lorentzian signature. The latter feature gives rise to the light-cone structure of spacetime, with vectors being divided into timelike, null or spacelike depending on whether $g(X, X)$ is negative, vanishing or positive, respectively. The classical laws of nature are written in tensor language, and gravity is the curvature of spacetime. In the theory of general relativity, gravity couples to the energy-momentum tensor of matter through the Einstein equations

$$
R_{a b}-\frac{1}{2} g_{a b} R=\frac{8 \pi G}{c^{4}} T_{a b}
$$

${ }^{\star}$ This paper is a contribution to the Proceedings of the 2007 Midwest Geometry Conference in honor of Thomas P. Branson. The full collection is available at http://www.emis.de/journals/SIGMA/MGC2007.html 
The Einstein-Hilbert action functional for gravity, giving rise to equation (1.1), is diffeomorphism-invariant, and hence general relativity belongs actually to the general set of theories ruled by an infinite-dimensional [28] invariance group (or pseudo-group). With hindsight, following DeWitt [31], one can say that general relativity was actually the first example of a non-Abelian gauge theory (about 38 years before Yang-Mills theory [98]).

\section{From Schrödinger to Feynman}

Quantum mechanics deals instead, mainly, with a description of the world on atomic or subatomic scale. It tells us that, on such scales, the world can be described by a Hilbert space structure, or suitable generalizations. Even in the relatively simple case of the hydrogen atom, the appropriate Hilbert space is infinite-dimensional, but finite-dimensional Hilbert spaces play a role as well. For example, the space of spin-states of a spin-s particle is $\mathbb{C}^{2 s+1}$ and is therefore finite-dimensional. Various pictures or formulations of quantum mechanics have been developed over the years, and their key elements can be summarized as follows:

(i) In the Schrödinger picture, one deals with wave functions evolving in time according to a first-order equation. More precisely, in an abstract Hilbert space $\mathcal{H}$, one studies the Schrödinger equation

$$
\mathrm{i} \hbar \frac{d \psi}{d t}=\hat{H} \psi
$$

where the state vector $\psi$ belongs to $\mathcal{H}$, while $\hat{H}$ is the Hamiltonian operator. In wave mechanics, the emphasis is more immediately put on partial differential equations, with the wave function viewed as a complex-valued map $\psi:(x, t) \rightarrow \mathbb{C}$ obeying the equation

$$
\mathrm{i} \hbar \frac{\partial \psi}{\partial t}=\left(-\frac{\hbar^{2}}{2 m} \triangle+V\right) \psi
$$

where $-\triangle$ is the Laplacian in Cartesian coordinates on $\mathbb{R}^{3}$ (with this sign convention, its symbol is positive-definite).

(ii) In the Heisenberg picture, what evolves in time are instead the operators, according to the first-order equation

$$
\mathrm{i} \hbar \frac{d \hat{A}}{d t}=[\hat{A}, \hat{H}]
$$

Heisenberg performed a quantum mechanical re-interpretation of kinematic and mechanical relations [65] because he wanted to formulate quantum theory in terms of observables only.

(iii) In the Dirac quantization, from an assessment of the Heisenberg approach and of Poisson brackets [32], one discovers that quantum mechanics can be made to rely upon the basic commutation relations involving position and momentum operators:

$$
\begin{aligned}
& {\left[\hat{q}^{j}, \hat{q}^{k}\right]=\left[\hat{p}_{j}, \hat{p}_{k}\right]=0,} \\
& {\left[\hat{q}^{j}, \hat{p}_{k}\right]=\mathrm{i} \hbar \delta_{k}^{j} .}
\end{aligned}
$$

For generic operators depending on $\hat{q}, \hat{p}$ variables, their formal Taylor series, jointly with application of (1.2) and (1.3), should yield their commutator. 
(iv) Weyl quantization. The operators satisfying the canonical commutation relations (1.3) cannot be both bounded, whereas it would be nice to have quantization rules not involving unbounded operators and domain problems. For this purpose, one can consider the strongly continuous one-parameter unitary groups having position and momentum as their infinitesimal generators. These read as $U(s) \equiv e^{\mathrm{i} s P}, V(t) \equiv e^{\mathrm{i} t Q}$, and satisfy the Weyl form of canonical commutation relations, which is given by

$$
U(s) V(t)=e^{\mathrm{i} s t} V(t) U(s) .
$$

Here the emphasis was, for the first time, on group-theoretical methods, with a substantial departure from the historical development, that relied instead heavily on quantum commutators and their relation with classical Poisson brackets.

(v) Feynman quantization (i.e., Lagrangian approach). The Weyl approach is very elegant and far-sighted, with several modern applications [47], but still has to do with a more rigorous way of doing canonical quantization, which is not suitable for an inclusion of relativity. A spacetime approach to ordinary quantum mechanics was instead devised by Feynman [49] (and partly Dirac himself [33]), who proposed to express the Green kernel of the Schrödinger equation in the form

$$
G\left[x_{f}, t_{f} ; x_{i}, t_{i}\right]=\int_{\text {all paths }} e^{\mathrm{i} S / \hbar} d \mu,
$$

where $d \mu$ is a suitable (putative) measure on the set of all space-time paths (including continuous, piecewise continuous, or even discontinuous paths) matching the initial and final conditions. This point of view has enormous potentialities in the quantization of field theories, since it preserves manifest covariance and the full symmetry group, being derived from a Lagrangian.

It should be stressed that quantum mechanics regards wave functions only as a technical tool to study bound states (corresponding to the discrete spectrum of the Hamiltonian operator $\hat{H}$ ), scattering states (corresponding instead to the continuous spectrum of $\hat{H}$ ), and to evaluate probabilities (of finding the values taken by the observables of the theory). Moreover, it is meaningless to talk about an elementary phenomenon on atomic (or sub-atomic) scale unless it is registered, and quantum mechanics in the laboratory needs also an external observer and assumes the so-called reduction of the wave packet (see [47] and references therein).

\section{Spacetime singularities}

Now we revert to the geometric side. In Riemannian or pseudo-Riemannian geometry, geodesics are curves whose tangent vector $X$ moves by parallel transport, so that eventually

$$
\frac{d X^{a}}{d s}+\Gamma_{b c}^{a} X^{b} X^{c}=0,
$$

where $s$ is the affine parameter and $\Gamma_{b c}^{a}$ are the connection coefficients. In general relativity, timelike geodesics correspond to the trajectories of freely moving observers, while null geodesics describe the trajectories of zero-rest-mass particles [63, Section 8.1]. Moreover, a spacetime $(M, g)$ is said to be singularity-free if all timelike and null geodesics can be extended to arbitrary values of their affine parameter. At a spacetime singularity in general relativity, all laws of classical physics would break down, because one would witness very pathological events such as the sudden disappearance of freely moving observers, and one would be completely unable to predict what came out of the singularity. In the sixties, Penrose [82] proved first an important theorem on the occurrence of singularities in gravitational collapse (e.g. formation of 
black holes). Subsequent work by Hawking [57, 58, 59, 60, 61], Geroch [50], Ellis and Hawking [62, 40], Hawking and Penrose [64] proved that spacetime singularities are generic properties of general relativity, provided that physically realistic energy conditions hold. Very little analytic use of the Einstein equations is made, whereas the key role emerges of topological and global methods in general relativity (jointly with Morse theory for Lorentzian manifolds).

\section{Unification of all fundamental interactions}

The fully established unifications of modern physics are as follows.

(i) Maxwell: electricity and magnetism are unified into electromagnetism. All related phenomena can be described by an antisymmetric rank-two tensor field, and derived from a one-form, called the potential.

(ii) Einstein: space and time are unified into the spacetime manifold. Moreover, inertial and gravitational mass, conceptually different, are actually unified as well.

(iii) Standard model of particle physics: electromagnetic, weak and strong forces are unified by a non-Abelian gauge theory, normally considered in Minkowski spacetime (this being the base space in fibre-bundle language).

The physics community is now familiar with a picture relying upon four fundamental interactions: electromagnetic, weak, strong and gravitational. The large-scale structure of the universe, however, is ruled by gravity only. All unifications beyond Maxwell involve non-Abelian gauge groups (either Yang-Mills or diffeomorphism group). Three extreme views have been developed along the years, i.e.,

(i) Gravity arose first, temporally, in the very early Universe, then all other fundamental interactions.

(ii) Gravity might result from Quantum Field Theory (this was the Sakharov idea [90]).

(iii) The vacuum of particle physics is regarded as a cold quantum liquid in equilibrium. Protons, gravitons and gluons are viewed as collective excitations of this liquid [95].

\section{From old to new unification}

Here we outline how the space-of-histories formulation provides a common ground for describing the 'old' and 'new' unifications of fundamental theories.

\section{Old unification}

Quantum field theory begins once an action functional $S$ is given, since the first and most fundamental assumption of quantum theory is that every isolated dynamical system can be described by a characteristic action functional [28]. The Feynman approach makes it necessary to consider an infinite-dimensional manifold such as the space $\Phi$ of all field histories $\varphi^{i}$. On this space there exist (in the case of gauge theories) vector fields

$$
Q_{\alpha}=Q_{\alpha}^{i} \frac{\delta}{\delta \varphi^{i}}
$$

that leave the action invariant, i.e., [31]

$$
Q_{\alpha} S=0 .
$$

The Lie brackets of these vector fields lead to a classification of all gauge theories known so far. 


\section{Type-I gauge theories}

The peculiar property of type-I gauge theories is that these Lie brackets are equal to linear combinations of the vector fields themselves, with structure constants, i.e., [30]

$$
\left[Q_{\alpha}, Q_{\beta}\right]=C_{\alpha \beta}^{\gamma} Q_{\gamma}
$$

where $C_{\alpha \beta, i}^{\gamma}=0$. The Maxwell, Yang-Mills, Einstein theories are all examples of type-I theories (this is the 'unifying feature'). All of them, being gauge theories, need supplementary conditions, since the second functional derivative of $S$ is not an invertible operator. After imposing such conditions, the theories are ruled by a differential operator of d'Alembert type (or Laplace type, if one deals instead with Euclidean field theory), or a non-minimal operator at the very worst (for arbitrary choices of gauge parameters). For example, when Maxwell theory is quantized via functional integrals in the Lorenz [74] gauge, one deals with a gauge-fixing functional

$$
\Phi(A)=\nabla^{b} A_{b}
$$

and the second-order differential operator acting on the potential reads as

$$
P_{a}^{b}=-\delta_{a}^{b} \square+R_{a}^{b}+\left(1-\frac{1}{\alpha}\right) \nabla_{a} \nabla^{b}
$$

where $\alpha$ is an arbitrary gauge parameter. The Feynman choice $\alpha=1$ leads to the minimal operator

$$
\widetilde{P}_{a}^{b}=-\delta_{a}^{b} \square+R_{a}^{b},
$$

which is the standard wave operator on vectors in curved spacetime. Such operators play a leading role in the one-loop expansion of the Euclidean effective action, i.e., the quadratic order in $\hbar$ in the asymptotic expansion of the functional ruling the quantum theory with positivedefinite metrics (see Section 6).

\section{Type-II gauge theories}

For type-II gauge theories, Lie brackets of vector fields $Q_{\alpha}$ are as in equation (2.2) for type-I theories, but the structure constants are promoted to structure functions. An example is given by simple supergravity (a supersymmetric $[53,96]$ gauge theory of gravity, with a symmetry relating bosonic and fermionic fields) in four spacetime dimensions, with auxiliary fields [78].

\section{Type-III gauge theories}

In this case, the Lie bracket (2.2) is generalized by

$$
\left[Q_{\alpha}, Q_{\beta}\right]=C_{\alpha \beta}^{\gamma} Q_{\gamma}+U_{\alpha \beta}^{i} S_{, i}
$$

and it therefore reduces to (2.2) only on the mass-shell, i.e., for those field configurations satisfying the Euler-Lagrange equations. An example is given by theories with gravitons and gravitinos such as Bose-Fermi supermultiplets of both simple and extended supergravity in any number of spacetime dimensions, without auxiliary fields [78]. 


\section{From supergravity to general relativity}

It should be stressed that general relativity is naturally related to supersymmetry, since the requirement of gauge-invariant Rarita-Schwinger equations [87] implies Ricci-flatness in four dimensions [27], which is then equivalent to vacuum Einstein equations. The Dirac operator [44] is more fundamental in this framework, since the $m$-dimensional spacetime metric is entirely re-constructed from the $\gamma$-matrices, in that

$$
g^{a b}=\frac{1}{2 m} \operatorname{tr}\left(\gamma^{a} \gamma^{b}+\gamma^{b} \gamma^{a}\right)
$$

\section{New unification}

In modern high energy physics, the emphasis is no longer on fields (sections of vector bundles in classical field theory, operator-valued distributions in quantum field theory), but rather on extended objects such as strings [26]. In string theory, particles are not described as points, but instead as strings, i.e., one-dimensional extended objects. While a point particle sweeps out a one-dimensional worldline, the string sweeps out a worldsheet, i.e., a two-dimensional real surface. For a free string, the topology of the worldsheet is a cylinder in the case of a closed string, or a sheet for an open string. It is assumed that different elementary particles correspond to different vibration modes of the string, in much the same way as different minimal notes correspond to different vibrational modes of musical string instruments [26]. The five different string theories [1] are different aspects of a more fundamental theory, called $M$-theory [9]. In the latest developments, one deals with 'branes', which are classical solutions of the equations of motion of the low-energy string effective action, that correspond to new non-perturbative states of string theory, break half of the supersymmetry, and are required by T-duality in theories with open strings. They have the peculiar property that open strings have their end-points attached to them [34,35]. With the language of pseudo-Riemannian geometry, branes are timelike surfaces embedded into bulk spacetime [7,6]. According to this picture, gravity lives on the bulk, while standard-model gauge fields are confined on the brane. For branes, the normal vector $N$ is spacelike with respect to the bulk metric $G_{A B}$, i.e.,

$$
G_{A B} N^{A} N^{B}=N_{C} N^{C}>0 .
$$

The action functional $S$ splits into the sum [6] $\left(g_{\alpha \beta}(x)\right.$ being the brane metric)

$$
S=S_{4}\left[g_{\alpha \beta}(x)\right]+S_{5}\left[G_{A B}(X)\right]
$$

while the effective action [30] $\Gamma$ is formally given by

$$
e^{\mathrm{i} \Gamma}=\int D G_{A B}(X) e^{\mathrm{i} S} \times \text { g.f. term. }
$$

In the functional integral, the gauge-fixed action reads as (here there is summation as well as integration over repeated indices $[28,30,6]$ )

$$
S_{\text {g.f. }}=S_{4}+S_{5}+\frac{1}{2} F^{A} \Omega_{A B} F^{B}+\frac{1}{2} \chi^{\mu} \omega_{\mu \nu} \chi^{\nu}
$$

where $F^{A}$ and $\chi^{\mu}$ are bulk and brane gauge-fixing functionals, respectively, while $\Omega_{A B}$ and $\omega_{\mu \nu}$ are non-singular 'matrices' of gauge parameters. The gauge-invariance properties of bulk and brane action functionals can be expressed by saying that there exist vector fields on the space of histories such that (cf. equation (2.1))

$$
R_{B} S_{5}=0, \quad R_{\nu} S_{4}=0
$$


whose Lie brackets obey a relation formally analogous to equation (2.2) for ordinary type-I theories, i.e.,

$$
\left[R_{B}, R_{D}\right]=C_{B D}^{A} R_{A}, \quad\left[R_{\mu}, R_{\nu}\right]=C_{\mu \nu}^{\lambda} R_{\lambda} .
$$

The bulk and brane ghost operators (see [29, 48] for the first time that ghost fields were considered in quantized gauge theories. When one studies how the gauge-fixing functional behaves under infinitesimal gauge transformations, one discovers that one can then define a differential operator acting on fields $\chi^{\beta}$ of the opposite statistics with respect to the fields occurring in the gauge-invariant action functional. Such fields $\chi^{\beta}$ are the ghost fields. They arise entirely from the fibre-bundle structure of the space of histories, from the Jacobian of the transformation from fibre-adapted coordinates to the conventional local fields.) are therefore

$$
Q_{B}^{A}=R_{B} F^{A}=F_{, a}^{A} R_{B}^{a}, \quad J_{\nu}^{\mu}=R_{\nu} \chi^{\mu}=\chi_{, i}^{\mu} R_{\nu}^{i},
$$

respectively. The full bulk integration means integrating first with respect to all bulk metrics $G_{A B}$ inducing on the boundary $\partial M$ the given brane metric $g_{\alpha \beta}(x)$, and then integrating with respect to all brane metrics. Thus, one first evaluates the cosmological wave function of the bulk spacetime [6], i.e.,

$$
\psi_{\mathrm{Bulk}}=\int_{G_{A B}[\partial M]=g_{\alpha \beta}} \mu\left(G_{A B}, S_{C}, T^{D}\right) e^{\mathrm{i} \widetilde{S}_{5}},
$$

where $\mu$ is taken to be a suitable measure, the $S_{C}, T^{D}$ are ghost fields, and

$$
\widetilde{S}_{5} \equiv S_{5}\left[G_{A B}\right]+\frac{1}{2} F^{A} \Omega_{A B} F^{B}+S_{A} Q_{B}^{A} T^{B} .
$$

Eventually, the effective action results from

$$
e^{\mathrm{i} \Gamma}=\int \widetilde{\mu}\left(g_{\alpha \beta}, \rho_{\gamma}, \sigma^{\delta}\right) e^{\mathrm{i} \widetilde{S}_{4}} \psi_{\mathrm{Bulk}},
$$

where $\widetilde{\mu}$ is another putative measure, $\rho_{\gamma}$ and $\sigma^{\delta}$ are brane ghost fields, and

$$
\widetilde{S}_{4} \equiv S_{4}+\frac{1}{2} \chi^{\mu} \omega_{\mu \nu} \chi^{\nu}+\rho_{\mu} J_{\nu}^{\mu} \sigma^{\nu}
$$

\section{Open problems}

Although string theory may provide a finite theory of quantum gravity that unifies all fundamental interactions at once, its impact on particle physics phenomenology and laboratory experiments remains elusive. Some key issues are therefore in sight:

(i) What is the impact (if any) of Planck-scale physics on cosmological observations [99]?

(ii) Will general relativity retain its role of fundamental theory, or shall we have to accept that it is only the low-energy limit of string or M-theory?

(iii) Are renormalization-group methods a viable way to do non-perturbative quantum gravity $[88,11]$, after the recent discovery of a non-Gaussian ultraviolet fixed point $[71,89,72]$ of the renormalization-group flow?

(iv) Is there truly a singularity avoidance in quantum cosmology [45, 46] or string theory $[68,66,67]$ ?

We are already facing unprecedented challenges, where the achievements of spacetime physics and quantum field theory are called into question. The years to come will hopefully tell us whether the new mathematical concepts considered in theoretical physics lead really to a better understanding of the physical universe and its underlying structures. 


\section{Mathematical promises and skepticism - the case of spectral geometry}

\section{The base of mathematical skepticism and promises}

When we write above of "unprecedented challenges, where the achievements of spacetime physics and quantum field theory are called into question" we are aware that large segments of the physics community actually are questioning the promised unified quantum gravity. We shall not repeat the physicists' skepticism which was skillfully gathered and elaborated, e.g., by Lee Smolin in [92].

Here we shall only add a skeptical mathematical voice, i.e., a remark made by Yuri Manin in a different context [76], elaborated in [77], and then try to draw a promising perspective out of Manin's remark.

The Closing round table of the International Congress of Mathematicians (Madrid, August 22-30, 2006) was devoted to the topic Are pure and applied mathematics drifting apart? As panelist, Manin subdivided the mathematization, i.e., the way mathematics can tell us something about the external world, into three modes of functioning (similarly Bohle, Booß and Jensen 1983, [10], see also [13]):

(i) An (ad-hoc, empirically based) mathematical model "describes a certain range of phenomena, qualitatively or quantitatively, but feels uneasy pretending to be something more". Manin gives two examples for the predictive power of such models, Ptolemy's model of epicycles describing planetary motions of about $150 \mathrm{BCE}$, and the standard model of around 1960 describing the interaction of elementary particles, besides legions of ad-hoc models which hide lack of understanding behind a more or less elaborated mathematical formalism of organizing available data.

(ii) A mathematically formulated theory is distinguished from an ad-hoc model primarily by its "higher aspirations. A theory, so to speak, is an aristocratic model." Theoretically substantiated models, such as Newton's mechanics, are not necessarily more precise than ad-hoc models; the coding of experience in the form of a theory, however, allows a more flexible use of the model, since its embedding in a theory universe permits a theoretical check of at least some of its assumptions. A theoretical assessment of the precision and of possible deviations of the model can be based on the underlying theory.

(iii) A mathematical metaphor postulates that "some complex range of phenomena might be compared to a mathematical construction". As an example, Manin mentions artificial intelligence with its "very complex systems which are processing information because we have constructed them, and we are trying to compare them with the human brain, which we do not understand very well - we do not understand almost at all. So at the moment it is a very interesting mathematical metaphor, and what it allows us to do mostly is to sort of cut out our wrong assumptions. If we start comparing them with some very well-known reality, it turns out that they would not work."

Clearly, Manin noted the deceptive formal similarity of the three ways of mathematization which are radically different with respect to their empirical foundation and scientific status. He expressed concern about the lack of distinction and how that may "influence our value systems". In the words of [13, p. 73]: "Well founded applied mathematics generates prestige which is inappropriately generalized to support these quite different applications. The clarity and precision of the mathematical derivations here are in sharp contrast to the uncertainty of the underlying relations assumed. In fact, similarity of the mathematical formalism involved tends to mask the differences in the scientific extra-mathematical status, in the credibility of the 
conclusions and in appropriate ways of checking assumptions and results ... Mathematization can - and therein lays its success - make existing rationality transparent; mathematization cannot introduce rationality to a system where it is absent ... or compensate for a deficit of knowledge."

Asked whether the last 30 years of mathematics' consolidation raise the chance of consolidation also in phenomenologically and metaphorically expanding sciences, Manin hesitated to use such simplistic terms. He recalled the notion of Kolmogorov complexity of a piece of information, which is, roughly speaking, "the length of the shortest programme, which can be then used to generate this piece of information ... Classical laws of physics - such phantastic laws as Newton's law of gravity and Einstein's equations - are extremely short programmes to generate a lot of descriptions of real physical world situations. I am not at all sure that Kolmogorov's complexity of data that were uncovered by, say, genetics in the human genome project, or even modern cosmology data ... is sufficiently small that they can be really grasped by the human mind."

In spite of our admiration of and sympathy with Manin's thoughtfulness, the authors of this review shall reverse Manin's argument and point to the astonishing shortness in the sense of Kolmogorov complexity of main achievements in one exemplary field of mathematics, in spectral geometry to encourage the new unification endeavor. Some of the great unifications in physics were preceded by mature mathematical achievements (like John Bernoulli's unification of light and particle movement after Leibniz' and Newton's infinitesimals and Einstein's general relativity after Riemann's and Minkowski's geometries). Other great unifications in physics were antecedent to comprehensive mathematical theory (like Maxwell's equations for electromagnetism long before Hodge's and de Rham's vector analysis of differential forms). A few great unifications in physics paralleled mathematical break-throughs (like Newton's unification of Kepler's planetary movement with Galilei's fall low paralleled calculus and Einstein's 1905 heat explanation via diffusion paralleled the final mathematical understanding of the heat equation via Fourier analysis, Lebesgue integral and the emerging study of Brownian processes).

In this section, we shall argue for our curiosity about the new unification, nourished by the remarkable shortness of basic achievements of spectral geometry and the surprisingly wide range of induced (inner-mathematical) explanations.

\section{The power of the index}

The most fundamental eigenvalue is the zero, and perhaps the most fundamental spectral invariant is the index of elliptic problems over compact manifolds without or with smooth boundary. It is the difference between the multiplicity of the zero eigenvalue of the original problem and of the adjoint problem. For a wide introduction to index theory we refer to [12] and [16], see also the more recent [14] and [44].

In this subsection we shall summarize the functional analytic framework of index theory (Fredholm pairs of subspaces, homotopy invariance of the index, classifying space), present simple formulations of the AS and APS theorems, recall how important geometric invariants can be written as index of corresponding elliptic problems, emphasize simple consequences for partitioned manifolds (cutting and pasting of the index) and delicate consequences for 4D geometry (Donaldson).

The basic object of index theory is a Fredholm pair $(M, N) \in \mathscr{F}^{2}$ of closed subspaces $M, N$ in a fixed Hilbert space $H$, i.e., $\operatorname{dim} M \cap N<\infty, M+N$ closed in $H$, and $\operatorname{dim} H /(M+N)<\infty$. We set

$$
\operatorname{ind}(M, N):=\operatorname{dim} M \cap N-\operatorname{dim} H /(M+N) .
$$

A bounded operator $F: H \rightarrow H, F \in \mathscr{B}$, is Fredholm, $F \in \mathscr{F}$, if the pair $(H \times\{0\}$, graph $F)$ is a Fredholm pair in $H \times H$, i.e., if $\operatorname{dim} \operatorname{ker} F<\infty$ and $\operatorname{dim}(H / \operatorname{im} F)<\infty$ such that im $F$ is 
closed and ind $F=\operatorname{dim} \operatorname{ker} F-\operatorname{dim} \operatorname{coker} F$. Note that then coker $F \equiv \operatorname{ker} F^{*}$, where $F^{*}$ denotes the adjoint operator to $F$.

The definition generalizes to closed (not necessarily bounded) operators, typically arising with elliptic differential operators, and to closed relations, typically arising in systems of differential equations when the set of singularities does not have measure zero.

It seems that up to 1950, D. Hilbert's and R. Courant's dictum was generally believed, that "linear problems of mathematical physics which are correctly posed" satisfy the so-called Fredholm alternative, i.e., their index vanishes. Then G. Hellwig and I. Vekua independently showed that the Laplace operator on the disc with a boundary condition given by a vector field of winding number $p$ has index $2-2 p$, i.e., $\neq 0$ for $p \neq 1$. A the same time, F.V. Atkinson gave the representation $\mathscr{F} \rightarrow(\mathscr{B} / \mathscr{K})^{\times} \rightarrow 0$, i.e., by the units of the quotient algebra of the bounded operators modulo the ideal $\mathscr{K}$ of compact operators (compact operators appear naturally with the inclusion of Sobolev spaces into each other); a little earlier, J. Dieudonné proved the homotopy invariance of the index, more precisely, that $\mathscr{F}$ decomposes in $\mathbb{Z}$ connected components, distinguished by the index. Later that was generalized by K. Jänich and M.F. Atiyah to a natural exact sequence of semigroups

$$
\left[X, \mathscr{B}^{\times}\right] \longrightarrow[X, \mathscr{F}] \stackrel{\text { ind }}{\longrightarrow} K(X) \longrightarrow 0 \quad \text { for any compact topological space } X \text {. }
$$

Here, $K(X)$ denotes the group of abstract difference classes of complex vector bundles over $X$ which was established by M.F. Atiyah and F. Hirzebruch as powerful cohomology theory satisfying Bott periodicity under suspension of $X ;[\cdot, \cdot]$ denotes the semigroup of homotopy classes from one space into another; and ind denotes the index bundle which, surprisingly, is well defined even when the dimension of the kernel of the Fredholm operators parametrized by $X$ varies. Note that then N. Kuiper proved that $\mathscr{B}^{\times}$is contractible, i.e., the vanishing of the left term in (4.1) yielding an isomorphism between $[X, \mathscr{F}]$ and $K(X)$. One says that $\mathscr{F}$ is a classifying space for the functor $K$. Setting $X:=$ \{point $\}$ brings (4.1) back to Dieudonné.

Similar results were obtained by I.M. Singer and M.F. Atiyah for the topology of the space $\mathscr{F}$ sa of self-adjoint bounded Fredholm operators: it consists of three connected components, two of them are contractible and the interesting component (those operators which are neither essentially positive nor essentially negative) is a classifying space for the functor $K^{1}$. For $X=S^{1}$, the isomorphism with $\mathbb{Z}$ is given by the spectral flow, see below.

Some of the results can be extended with minor modifications to the spaces $\mathscr{C} \mathscr{R} \mathscr{F}$ of closed Fredholm relations, $\mathscr{C} \mathscr{F}$ (closed Fredholm operators) or $\mathscr{C} \mathscr{F}$ sa (self-adjoint, not necessarily bounded Fredholm operators).

The interest in the index was nourished by the question (I.M. Singer): "Why are so many geometric invariants integer valued, like the $\widehat{A}$ genus of spin manifolds which is given a priori as an integral?" The answer, Singer and Atiyah found in 1962, see [2], is that $\widehat{A}$ and many other important geometric invariants like Euler characteristic and Thom-Hirzebruch signature can be written as the index of an elliptic differential operator. This is the essence of the celebrated Atiyah-Singer Index Theorem. We give a simplified version: Let $M$ be a closed (i.e., compact, without boundary), oriented, smooth Riemannian manifold of dimension $n$, which is "trivially" embedded (i.e., with trivial normal bundle) in the Euclidean space $\mathbb{R}^{n+m}$. Let $E$ and $F$ be Hermitian vector bundles over $M$ and $A$ a linear elliptic operator of order $k \in \mathbb{Z}$, mapping sections of $E$ to sections of $F$. Its leading symbol $\sigma(A)^{1}$ is a bundle isomorphism from $E$ to $F$ lifted to the cotangent sphere bundle $S(M)$ and defines an element $[\sigma(A)]$ in the abstract ring

\footnotetext{
${ }^{1}$ Recall that for a differential operator $D=\sum_{\alpha \in \mathbb{Z}_{+}^{n}, \alpha_{1}+\cdots+\alpha_{n} \leq d} a_{\alpha}(x) \partial_{1}^{\alpha_{1}} \cdots \partial_{n}^{\alpha_{n}}$ the leading symbol at the cotangent vector $\xi_{i} d x^{i}$ is given by $\sum_{\alpha \in \mathbb{Z}_{+}^{n}, \alpha_{1}+\cdots+\alpha_{n}=d} a_{\alpha}(x) \xi_{1}^{\alpha_{1}} \cdots \xi_{n}^{\alpha_{n}}$. The leading symbol has an invariant meaning
} as a section of the bundle $\operatorname{Hom}(E, F)$ over $T^{*} M$. 
$K\left(T^{*} M\right)$ of formal differences of vector bundles over the full cotangent bundle $T^{*} M$ of $M$. Then

$$
\text { ind } A=(-1)^{n} \beta^{n+m}\left([\sigma(A)] \otimes b^{m}\right) \text {, }
$$

where $b \in K\left(\mathbb{R}^{2}\right)$ denotes the Bott class (a generator of $K\left(\mathbb{R}^{2}\right) \simeq \mathbb{Z}$ ) and $\beta^{n+m}: K\left(\mathbb{R}^{2(n+m)}\right) \rightarrow \mathbb{Z}$ the iteration of the Bott isomorphism.

(4.2) comprises among numerous other applications Gauss-Bonnet's expression of the Euler characteristic by a curvature integral, Hirzebruch's formula of the signature by $L$-polynomials, and the celebrated Riemann-Roch-Hirzebruch Theorem for complex manifolds. A special feature of (4.2) is that we have on the left something which is globally defined, namely by the multiplicity of the zero-eigenvalues, whereas on the right we have an expression which is defined by the leading symbol of the operator $A$, i.e., in terms of the coefficients of the operator. Actually, the right hand side can conveniently be written as $\int_{M} \alpha_{0}(x) d x$ where $\alpha_{0}(x)$ is the index density. It is the constant term in the asymptotic expansion (as $t \rightarrow 0$ ) of the trace differences

$$
\sum_{\mu \in \operatorname{spec} A A^{*}} e^{-t \mu}\left|\phi_{\mu}(x)\right|^{2}-\sum_{\mu^{\prime} \in \operatorname{spec} A^{*} A} e^{-t \mu^{\prime}}\left|\phi_{* \mu^{\prime}}(x)\right|^{2}
$$

where $\mu, \phi_{\mu}$ denote the eigenvalues and eigenfunctions of $A A^{*}$ and $\mu^{\prime}, \phi_{* \mu^{\prime}}$ the corresponding objects of $A^{*} A$.

A simple model of the Index Theorem is provided by the winding number $\operatorname{deg}(f)$ of a continuous mapping $f: S^{1} \rightarrow \mathbb{C}^{\times}$of the circle to the punctured plane of non-zero complex numbers. We approximate $f$ by a differentiable $g$ or by a finite Laurent series $h(z)=\sum_{\nu=-k}^{k} a_{v} z^{\nu}$ on the disk $|z|<1$ yielding

$$
\operatorname{deg}(f)=N(h)-P(h)=\frac{1}{2 \pi \mathrm{i}} \int \frac{d g}{g},
$$

where $N$ and $P$ denote the number of zeros and poles in $|z|<1$. A direct relation to (4.2) is that $\operatorname{deg}(f)=-\operatorname{ind} T_{f}$ where $T_{f}$ denotes the Toeplitz operator ${ }^{2}$, assigned to $f$ on the halfspace $L_{+}^{2}\left(S^{1}\right)$ spanned by the functions $z^{0}, z^{1}, z^{2}, \ldots$

Many quite different proofs of (4.2) have been given. At the short end of the scale, regarding the length of proof, is reducing all cases to (elliptic) Dirac operators and simple manifolds by cobordism (à la Hirzebruch) or reducing all cases to the winding number calculation plus Bott periodicity by embedding in huge Euclidean space (à la Grothendieck). On the long end (cf. [3, p. 281]) of the scale and promising more insight in the underlying geometry are the heat equation proofs, inspired by (4.3) (à la Minakshisundaram and Pleijel).

To obtain a Fredholm operator from an elliptic operator $A$ over a compact manifold $M$ with boundary $\Sigma$ one has to impose suitable boundary conditions. They can be locally defined by bundle homomorphisms or globally by pseudodifferential projections like the positive spectral (Atiyah-Patodi-Singer-) projection $P_{+}{ }^{3}$ of the tangential operator $B$, if $A$ is of Dirac type. Then (4.2) generalizes to

$$
\text { ind } A_{P_{+}}=\int_{M} \alpha_{0}(x) d x-\frac{\operatorname{dim} \operatorname{ker}(B)+\eta(0)}{2},
$$

where $\eta(0)$ denotes the $\eta$ invariant of $B$, see below. Note that $\operatorname{dim}$ ker $B$ can jump, e.g., under continuous deformation of the Riemannian metric. Thus, ind $A_{P_{+}}$is not homotopy invariant

\footnotetext{
${ }^{2}$ In general a Toeplitz operator is the compression of a multiplication operator by a projection, i.e. $P M_{f} P$. Here $T_{f}=P\{$ multiplication by $f\} P$, where $P$ is the orthogonal projection onto $L_{+}^{2}\left(S^{1}\right)$.

${ }^{3}$ i.e. the orthogonal projection onto the subspace spanned by the eigenvectors to nonnegative eigenvalues of $B$.
} 
(under homotopies of the metric) in spite of being the index of a Fredholm operator. Of course this does not contradict Dieudonné's theorem: $P_{+}$does not (necessarily) depend continuously on the metric and hence so does not the operator $A_{P_{+}}$. Consequently, a continuous change of the coefficients or the underlying metric structures can result in a jump of the Fredholm operator from one connected component into another one.

The Atiyah-Patodi-Singer boundary value problem is an example of a well-posed elliptic boundary value problem. We elaborate a bit on such boundary value problems for a (total hence formally self-adjoint) Dirac operator $A$. A boundary condition for $A$ is given by a pseudodifferential operator $P$ of order 0 . It is not a big loss of generality to assume that $P$ is an idempotent. The domain $\mathscr{D}\left(A_{P}\right)$ of the realization $A_{P}$ then consists of those sections $u$ (of Sobolev class 1) such that $P\left(u_{\mid \Sigma}\right)=0$. Looking at Green's formula for $A$

$$
\langle A u, v\rangle-\langle u, A v\rangle=-\int_{\Sigma}\langle\gamma u, v\rangle d \mathrm{vol}=: \omega\left(u_{\mid \Sigma}, v_{\mid \Sigma}\right)=\left\langle\gamma u_{\mid \Sigma}, v_{\mid \Sigma}\right\rangle,
$$

where $\gamma$ is Clifford multiplication by the inward normal vector, we see that the right hand side of (4.5) equips $L^{2}\left(S_{\mid \Sigma}\right)$ with a natural Hermitian symplectic structure. It turns out that a realization $A_{P}$ of a well-posed elliptic boundary value problem $P$ is self-adjoint if and only if the range of $P$ is Lagrangian in $\left(L^{2}\left(S_{\mid \Sigma}\right), \omega\right)$. Well-posedness is originally a microlocal condition. However, for Lagrangian $P$ it is equivalent to the fact that the pair of subspaces $\left(\operatorname{ker} P, \operatorname{im} P_{+}\right)$ is Fredholm [20].

On partitioned manifolds $M=M_{-} \cup_{\Sigma} M_{+}$one has the Bojarski Formula

$$
\text { ind } A=\operatorname{ind}\left(H_{-}(A), H_{+}(A)\right) \text {, }
$$

where $\left(H_{-}(A), H_{+}(A)\right)$ denotes the Fredholm pair of Cauchy data spaces (=boundary values of solutions to the equation $A u=0$ on $M_{+}$respectively $M_{-}$) along the separating hypersurface $\Sigma$. Contrary to the precise Novikov additivity of Euler characteristic and signature under cutting and pasting, decomposition formulas for all other indices contain non-vanishing corrections in terms of the geometry of $\Sigma$ and the gluing diffeomorphisms.

Novikov additivity of Euler characteristic and signature can be explained by the index theorem, but, being combinatorial, is more easily obtained by purely topological arguments. Some deep insight in the geometry of 3- and 4-dimensional differentiable manifolds, however, seems to rely on the index theorem. Most famous is S. Donaldson's theorem [36] that any closed smooth simply-connected 4-manifold $M$ with positive-definite intersection form $s_{M}$ can be written as the boundary of a 5-manifold, is a connected sum of complex projective spaces and $s_{M}$ is trivial, i.e., in standard diagonal form. The proof depends on the investigation of the moduli space of solutions of the Yang-Mills equation of gauge-theoretic physics. Note that by a famous theorem of M. Freedman any unimodular (integral) quadratic form can appear as $s_{M}$ for exactly one or two simply-connected oriented topological 4-manifolds. By that Freedman confirmed Poincaré's Conjecture in dimension 4. Moreover, according to Freedman there exist $10^{51} \mathrm{mu}-$ tually non homeomorphic oriented simply-connected topological manifolds with 40-dimensional 2-homology (i.e., with 40 bubbles), but, according to Donaldson, only one (more precisely two) permit a differentiable structure. Note that all piecewise linear 4-manifolds can be equipped with a differentiable structure, i.e., the difference between differentiable and solely topological cannot be perceived by appealing to piecewise linear constructions.

As another consequence of Donaldson's theorem, $\mathbb{R}^{4}$ admits an exotic differentiable structure which renders it not diffeomorphic to $\mathbb{R}^{4}$ with its usual differentiable structure.

\section{Other spectral invariants}

While the index, e.g., of the chiral Dirac operator $A^{+}$only measures the (chiral) asymmetry of the zero eigenspaces, there are three other spectral invariants which can provide much more 
information about a geometry one may be interested in: the spectral flow, the $\eta$ invariant and the determinant.

The spectral flow sf measures the net sign change of the eigenvalues around zero for a curve in $\mathscr{C} \mathscr{F}^{\mathrm{sa}}$ (cf. the previous section). For fixed endpoints, it does not change under a homotopy of the curve. For bounded self-adjoint (and neither essentially positive nor essentially negative) Fredholm operators, the spectral flow yields an isomorphism of the fundamental group on the integers. Only recently [69] it was shown that the same statement also holds for $\mathscr{C} \mathscr{F}$ sa equipped with the graph (=gap) topology. Surprisingly, the latter space is connected as was observed first in [15].

Referring to (4.6) (which is for differential operators only meaningful on even-dimensional manifolds), we consider a curve of (total) Dirac operators over a partitioned manifold. Then the Cauchy data spaces along the separating hypersurface $\Sigma$ are Fredholm pairs of Lagrangian subspaces of the symplectic Hilbert space $L^{2}\left(\Sigma,\left.S\right|_{\Sigma}\right)(\mathrm{cf} .(4.5))$ and we have

$$
\operatorname{sf}\left\{A_{s}\right\}_{0 \leq s \leq 1}=\operatorname{Mas}\left\{\left(H_{-}\left(A_{s}\right), H_{+}\left(A_{s}\right)\right)\right\}_{0 \leq s \leq 1},
$$

where Mas denotes the Maslov index, which adds the (finite and signed) intersection dimensions. Note that the left side of (4.7) is defined by the spectrum (actually, a generalization of the Morse index), while the right side is more "classical" and, in general, easier to calculate (actually, a generalization of the number of conjugate points). Ongoing research shows that formula (4.7) can be obtained in much greater generality.

Note also that we have ind $\left(\frac{d}{d s}+B_{s}\right)=\operatorname{sf}\left\{B_{s}\right\}$ for any curve of elliptic operators of first order over a closed manifold $\Sigma$ with unitarily equivalent ends, when the operator on the left side is induced on the corresponding mapping torus.

It turns out, that not only the chiral and the dynamic asymmetry of the zero eigenspaces can be measured, but also the asymmetry of the total spectrum of an operator $A$ of Dirac type on a closed manifold. Thus, roughly speaking, we have a formally self-adjoint operator with a real discrete spectrum which is nicely spaced without finite accumulation points and with an infinite number of eigenvalues on both sides of the real line. In close analogy with the definition of the zeta function for essentially positive elliptic operators like the Laplacian, we set

$$
\eta(z):=\sum_{\lambda \in \operatorname{spec}(A) \backslash\{0\}} \operatorname{sign}(\lambda) \lambda^{-z}=\frac{1}{\Gamma\left(\frac{z+1}{2}\right)} \int_{0}^{\infty} t^{\frac{z-1}{2}} \operatorname{Tr}\left(A e^{-t A^{2}}\right) d t .
$$

Clearly, the formal sum $\eta(z)$ is well defined for complex $z$ with $\operatorname{Re}(z)$ sufficiently large, and it vanishes for a symmetric spectrum.

For comparison we give the corresponding formula for the zeta function of the (positive) Dirac Laplacian $A^{2}$ :

$$
\zeta_{A^{2}}(z):=\operatorname{Tr}\left(\left(A^{2}\right)^{-z}\right)=\frac{1}{\Gamma(z)} \int_{0}^{\infty} t^{z-1} \operatorname{Tr}\left(e^{-t A^{2}}\right) d t .
$$

For the zeta function, we must assume that $A$ has no vanishing eigenvalues (i.e., $A^{2}$ is positive). Otherwise the integral on the right side is divergent. (The situation, however, can be cured by subtracting the orthogonal projection onto the kernel of $A^{2}$ from the heat operator before taking the trace.) For the eta function, on the contrary, it clearly does not matter whether there are 0 -eigenvalues and whether the summation is over all or only over the nonvanishing eigenvalues.

The derivation of (4.8) and (4.9) is completely elementary for $\operatorname{Re}(z)>\frac{1+\operatorname{dim} M}{2}, \operatorname{resp} . \operatorname{Re}(z)>$ $\frac{\operatorname{dim} M}{2}$, where $M$ denotes the underlying manifold. Next, recall the heat trace expansion of elliptic operators which holds in great generality:

$$
\operatorname{Tr}\left(B e^{-t T}\right) \sim_{t \rightarrow 0+} \sum_{j=0}^{\infty} a_{j}(T, B) t^{(j-\operatorname{dim} M-b) / 2}+\sum_{j=0}^{\infty}\left(b_{j}(T, B)+c_{j}(T, B) \log t\right) t^{j},
$$


$b=$ order of $B$, for any (pseudo)differential operator $B$, and any positive definite self-adjoint elliptic (pseudo)differential operator $T$ (here for simplicity of order 2) [55].

Using (4.10), it follows at once that $\eta(z)$ (and $\zeta(z)$ ) admit a meromorphic extension to the whole complex plane. However, a priori 0 is a pole. In general the residue at 0 (which is a multiple of the coefficient of $\log t$ in (4.10)) of the generalized $\zeta$-function $\operatorname{Tr}\left(B T^{-z}\right)=$ $\frac{1}{\Gamma(z)} \int_{0}^{\infty} t^{z-1} \operatorname{Tr}\left(B e^{-t T}\right) d t$ turns out to be an invariant of $B$ independently of $T$ : it is the celebrated non-commutative residue $[43,41,42,21]$ discovered independently by V. Guillemin [56] and M. Wodzicki [97]. The residue of Id is easily seen to vanish. Consequently the $\zeta$-function (4.9) is always regular at 0 . In fact Wodzicki proved that the residue of any (pseudo)differential idempotent vanishes and from this one can conclude that also the $\eta$-function (4.8) is always regular at 0 .

In the decade or so following 1975, it was generally believed that the existence of a finite eta invariant was a very special feature of operators of Dirac type on those closed manifolds which are boundaries. Later Gilkey [52] proved that the eta-function is regular at 0 for any self-adjoint elliptic operator on a closed manifold, cf. also Branson and Gilkey [17]. Only after the seminal paper by Douglas and Wojciechowski [37] it was gradually realized that globally elliptic self-adjoint boundary value problems for operators of Dirac type also have a finite eta invariant. Today, there are quite different approaches to obtain that result.

Historically, the eta invariant appeared for the first time in the 1970s as an error term showing up in the index formula for the APS spectral boundary value problem of a Dirac operator $A$ on a compact manifold $M$ with smooth boundary $\Sigma$ (see above (4.4)). More precisely, what arose was the eta invariant of the tangential operator (i.e., the induced Dirac operator over the closed manifold $\Sigma$ ). Even in that case it was hard to establish the existence and finiteness of the eta invariant.

The eta-invariant and the spectral flow are intimately related: let $A_{s}$ be a smooth family of Dirac operators on a closed manifold. Then $\xi\left(A_{s}\right):=\frac{1}{2}\left(\operatorname{dim} \operatorname{ker} A_{s}+\eta\left(A_{s}\right)\right)$ has only integer jumps and hence the reduced eta-invariant $\widetilde{\eta}\left(A_{s}\right):=\left(\frac{1}{2}\left(\operatorname{dim} \operatorname{ker} A_{s}+\eta\left(A_{s}\right)\right) \bmod \mathbb{Z}\right) \in \mathbb{R} / \mathbb{Z}$ varies smoothly. The variation of the reduced eta-invariant is known to be local:

$$
\frac{d}{d s} \widetilde{\eta}\left(A_{s}\right)=-\frac{1}{\sqrt{\pi}} \operatorname{Pf}_{t \rightarrow 0+} \sqrt{t} \operatorname{Tr}\left(\dot{A}_{s} e^{-t A_{s}^{2}}\right)
$$

where Pf (partie finie) is a short hand for the coefficient of $t^{0}$ in the asymptotic expansion. Because of the integer jumps of $\xi\left(A_{s}\right)$ the fundamental theorem of calculus now reads

$$
\eta\left(A_{1}\right)-\eta\left(A_{0}\right)=2 \operatorname{sf}\left\{A_{s}\right\}+2 \int_{0}^{1} \frac{d}{d s} \widetilde{\eta}\left(A_{s}\right) d s .
$$

It is a matter of convention how to count 0 -modes of $A_{0}, A_{1}$ for the spectral flow and we ignore it here.

Unlike the index and spectral flow on closed manifolds, we have neither an established functional analytical nor a topological frame for discussing $\eta(0)$, nor is it given by an integral of a locally defined expression. On the circle, e.g., consider the operator

$$
\mathcal{D}_{a}:=-\mathrm{i} \frac{d}{d x}+a=e^{-\mathrm{i} x a} \mathcal{D}_{0} e^{\mathrm{i} x a},
$$

so that $\mathcal{D}_{a}$ and $\mathcal{D}_{0}$ are locally unitarily equivalent, but not globally. In particular, $\eta_{\mathcal{D}_{a}}(0)=-2 a$ depends on $a$.

The reduced $\eta$-invariant depends, however, only on finitely many terms of the complete symbol of $A$.

By the Cobordism Theorem, the index vanishes on any closed (odd-dimensional) manifold $\Sigma$ for any elliptic operator which is the chiral tangential operator of a Dirac type operator on 
a suitable (even-dimensional) manifold which has $\Sigma$ as its boundary. This can be explained by the vanishing of the induced symplectic form over $\Sigma$. Correspondingly, we have in even dimensions, that the eta invariant vanishes on any closed manifold $\Sigma$ for any elliptic operator which is the tangential operator of a Dirac type operator on a suitable manifold which has $\Sigma$ as its boundary. This can be explained by the induced precise symmetry of the spectrum due to the anti-commutativity of the tangential operator with Clifford multiplication.

To us, the eta invariant of a Dirac type operator can be best understood as the phase of the zeta regularized determinant. But what is the determinant of an operator with an infinite number of eigenvalues?

From the point of view of functional analysis, the only natural concept is the Fredholm determinant of operators of the form $e^{\alpha}$ or, more generally, $\mathrm{Id}+\alpha$ where $\alpha$ is of trace class. We recall the formulas

$$
\operatorname{det}_{\mathrm{Fr}} e^{\alpha}=e^{\operatorname{Tr} \alpha} \quad \text { and } \quad \operatorname{det}_{\mathrm{Fr}}(\mathrm{Id}+\alpha)=\sum_{k=0}^{\infty} \operatorname{Tr} \wedge^{k} \alpha .
$$

The Fredholm determinant is notable for obeying the product rule, unlike other generalizations of the determinant to infinite dimensions where the error of the product rule leads to new invariants.

Clearly, the parametrix of a Dirac operator leads to operators for which the Fredholm determinant can be defined, but the relevant information about the spectrum of the Dirac operator does not seem sufficiently maintained. Note also that Quillen and Segal's construction of the determinant line bundle is based on the concept of the Fredholm determinant, though without leading to a number when the bundle is non-trivial.

Another concept is the $\zeta$-function regularized determinant, based on Ray and Singer's observation that, formally,

$$
\operatorname{det} T=\prod \lambda_{j}=\exp \left\{\left.\sum \ln \lambda_{j} e^{-z \ln \lambda_{j}}\right|_{z=0}\right\}=e^{-\left.\frac{d}{d z} \zeta_{T}(z)\right|_{z=0}}
$$

where $\zeta_{T}(z):=\sum_{j=1}^{\infty} \lambda_{j}^{-z}=\frac{1}{\Gamma(z)} \int_{0}^{\infty} t^{z-1} \operatorname{Tr} e^{-t T} d t$ as in (4.9). As explained before, for a positive definite self-adjoint elliptic operator $T$ of second order, acting on sections of a Hermitian vector bundle over a closed manifold $M$, the function $\zeta_{T}(z)$ is holomorphic for $\operatorname{Re}(z)$ sufficiently large and can be extended meromorphically to the whole complex plane with $z=0$ no pole.

The previous definition does not apply immediately to the Dirac operator $A$ which has infinitely many positive eigenvalues $\lambda_{j}$ and negative eigenvalues $-\mu_{j}$. As an example, consider again the operator $\mathcal{D}_{a}$ with $\operatorname{spec} \mathcal{D}_{a}=\{k+a\}_{k \in \mathbb{Z}}$.

On choosing the branch $(-1)^{-z}=e^{\mathrm{i} \pi z}$, we find

$$
\zeta_{A}(z)=\sum \lambda_{j}^{-z}+\sum(-1)^{-z} \mu_{j}^{-z}=\frac{1}{2}\left\{\zeta_{A^{2}}\left(\frac{z}{2}\right)+\eta_{A}(z)\right\}+\frac{1}{2} e^{\mathrm{i} \pi z}\left\{\zeta_{A^{2}}\left(\frac{z}{2}\right)-\eta_{A}(z)\right\},
$$

where $\eta_{A}(z)$ is defined as in (4.8). Thus:

$$
\zeta_{A}^{\prime}(0)=\frac{1}{2} \zeta_{A^{2}}^{\prime}(0)+\frac{\mathrm{i} \pi}{2}\left\{\zeta_{A^{2}}(0)-\eta_{A}(0)\right\} \quad \text { and } \quad \operatorname{det}_{\zeta} A=e^{-\zeta_{A}^{\prime}(0)}
$$

This relation between the phase of the $\zeta$-determinant and the $\eta$-invariant does in fact hold in great generality, at least for self-adjoint elliptic operators on closed manifolds.

There are a few classes of operators for which the $\zeta$-determinant can be calculated quite explicitly. The most satisfying theory is available for one-dimensional operators. Consider a onedimensional Schrödinger operator on the interval $[0,1]$ with, to make things a bit interesting, 
a regular singular potential

$$
L:=-\frac{d^{2}}{d x^{2}}+V(x), \quad V(x)=\frac{q(x)}{x^{2}(1-x)^{2}},
$$

with some smooth function $q(x), 0 \leq x \leq 1, q(0), q(1) \geq-1 / 4$. The last condition guarantees that $L$ is bounded below and thus we can consider the Friedrichs extension $L^{\mathscr{F}}$. Physically this means that the potential walls at 0,1 are infinitely high and hence the quantum-mechanical particle is trapped in the interval $[0,1]$. Then the $\zeta$-determinant of $L^{\mathscr{F}}$ can be expressed in terms of the fundamental solutions of the homogeneous differential equation $L f=0$ (see [73], also for a history of the problem). Indeed, choose solutions $\varphi, \psi$ of this equation satisfying $\varphi(x) \sim_{x \rightarrow 0} x^{\nu_{0}+1 / 2}, \psi(x) \sim_{x \rightarrow 0}(1-x)^{\nu_{1}+1 / 2}, \nu_{j}=\sqrt{a(j)+1 / 4}, j=0,1$. Then

$$
\operatorname{det}_{\zeta}\left(L^{\mathscr{F}}\right)=\frac{\pi\left(\psi \varphi^{\prime}-\varphi \psi^{\prime}\right)}{2^{\nu_{0}+\nu_{1}} \Gamma\left(\nu_{0}+1\right) \Gamma\left(\nu_{1}+1\right)} .
$$

Note that the Wronskian $\psi \varphi^{\prime}-\varphi \psi^{\prime}$ is a constant.

This formula can to a certain extent be generalized to higher dimensions. Note that the solutions of the homogeneous differential equation are determined by their Cauchy data at the boundary. Therefore the Wronskian should be viewed as a Fredholm determinant ${ }^{4}$ on the boundary.

For Dirac operators, S. Scott and K.P. Wojciechowski [91] found an exciting relation between the Fredholm determinant and the zeta-regularized determinant: Let $A$ be a Dirac operator over a compact manifold $M$ with boundary $\Sigma$ and tangential operator $\left(\begin{array}{cc}0 & B^{-} \\ B^{+} & 0\end{array}\right)$. The decomposition of the tangential operator is induced by the \pm i-eigenbundle decomposition of the natural symplectic structure (cf. (4.5)); in the odd-dimensional case this is the chiral decomposition on the even-dimensional boundary. Let $P$ be the APS or another suitable pseudodifferential projection defining a regular elliptic boundary value problem for $A$. Then the Cauchy data space $H_{+}(A)$ (i.e., the range of the Calderón projection $C_{+}$) and the range of $P$ can be written as the graphs of unitary, elliptic operators of order $0, K$, resp. $T$ which differ from the operator $\left(B^{+} B^{-}\right)^{-1 / 2} B^{+}: C^{\infty}\left(\Sigma ;\left.S^{+}\right|_{\Sigma}\right) \rightarrow C^{\infty}\left(\Sigma ;\left.S^{-}\right|_{\Sigma}\right)$ by a smoothing operator. Then

$$
\operatorname{det}_{\zeta} A_{P}=\operatorname{det}_{\zeta} A_{C_{+}} \cdot \operatorname{det}_{\mathrm{Fr}} \frac{1}{2}\left(\mathrm{I}+K T^{-1}\right) \text {. }
$$

This formula shows how the $\zeta$-determinant depends on the choice of the boundary condition. As in the one-dimensional case, the space of solutions of the homogeneous operator equation $A u=0$, through its Calderón projector, enters crucially.

Finally we mention another class of operators where relative $\zeta$-determinants can be calculated: let $M$ be a closed oriented surface with a Riemannian metric $g$. Let $h$ be a smooth function on $M$ and consider the conformally changed metric $g_{h}=e^{2 h} g$. By $\Delta_{h}$ we denote the (positive definite) Laplacian with respect to the metric $g_{h}$. Then the Polyakov formula reads

$$
\log \frac{\operatorname{det}_{\zeta} \Delta_{h}}{\operatorname{det}_{\zeta} \Delta_{0}}=-\frac{1}{12 \pi} \int_{M}|\nabla h|^{2} \operatorname{dvol}_{0}-\frac{1}{6 \pi} \int_{M} K_{0} h \operatorname{dvol}_{0}+\log \frac{\operatorname{vol}\left(M, g_{0}\right)}{\operatorname{vol}\left(M, g_{h}\right)} .
$$

Here $\nabla$ is the Levi-Civita connection and $K_{0}$ is the Gaussian curvature of the metric $g_{0}$. The formula (4.11) goes back to Polyakov [83, 84]. It was used in the work of Osgood, Phillips, and Sarnak [79] who showed that exactly the constant curvature metrics are the extremals of the determinant in a conformal class. More interestingly, they did this without using the Uniformization Theorem. Rather their method reproves this theorem.

\footnotetext{
${ }^{4}$ Here it is a finite-dimensional determinant, since the boundary is 0-dimensional and hence the space of sections on the boundary is finite-dimensional.
} 
Formulas similar to (4.11) were obtained by T. Branson and B. Ørsted for the conformal Laplacian in four dimensions [18].

Finally we would like to provide a glimpse at pasting formulas for $\eta$-invariants and $\zeta$ determinants since in the last decade substantial progress has been made. Compared to pasting formulas for the index (cf. (4.6), Novikov additivity) this is technically much more involved. The case which is most similar to the Euler characteristic is that of the analytic torsion. Using the celebrated Cheeger-Müller theorem on the relation between analytic and combinatorial torsion one obtains pasting formulas more or less by employing the doubling trick: on the double $\widetilde{M}=M \bigcup_{\partial M} M$ differential forms split naturally into a sum of forms obeying the relative resp. absolute boundary conditions on $\partial M[75]$. A direct analytic approach is also possible but technically much more involved [94]. It was Vishik's great idea to consider on a partitioned manifold $M_{1} \cup_{\Sigma} M_{2}$ the following family of boundary conditions for differential forms

$$
\sin \Theta i_{1}^{*} \omega=\cos \Theta i_{2}^{*} \omega,
$$

where $i_{j}$ is the pullback to $\Sigma$ from $M_{j}$. This family interpolates between the direct sum of the absolute boundary condition on $M_{1}$ and the relative boundary condition on $M_{2}$ on the one hand $(\Theta=0)$ and the continuous transmission condition on $\Sigma(\Theta=\pi / 4)$ on the other hand.

This motive was later adapted to Dirac operators and the $\eta$-invariant [19]. The final gluing formula for the $\eta$-invariant of a Dirac operator on a partitioned manifold reads

$$
\eta(D)-\eta\left(D_{P} \mid M_{2}\right)-\eta\left(D_{I-P} \mid M_{1}\right)=\operatorname{Mas}\left(P_{1}, P, I-P_{2}\right) .
$$

Here, $P$ is a well-posed Lagrangian boundary condition on $M_{1}$ as explained after (4.5) and $\operatorname{Mas}\left(P_{1}, P, I-P_{2}\right)$ is a Maslov type triple index involving the boundary condition $P$ and the Calderón projectors (projectors on the Cauchy data spaces) on $M_{1}, M_{2}$ [70].

In a titanic effort Park and Wojciechowski $[80,81]$ eventually succeeded to prove an analytic surgery formula for the $\zeta$-determinant. We refrain from giving a precise formulation, see loc. cit.

\section{Spectral triples and other new ideas and results of noncommutative geometry}

Noncommutative Geometry is an area of mathematics which has been dominated by the work of Alain Connes over the last 20-25 years. The basic idea is that instead of point sets (e.g. manifolds) one studies the coordinate ring of (smooth) functions. This point of view has been around in algebraic geometry for decades but it was Connes who showed that also manifolds and index theory can be understood from this perspective.

The starting point of Noncommutative Topology is the well-known Gelfand-representation theorem for commutative $C^{*}$-algebras: to any compact space $X$ we can associate its $\operatorname{ring} C(X)$ of continuous functions. This is a commutative $C^{*}$-algebra. Conversely, if $\mathcal{A}$ is a commutative $C^{*}$ algebra then we can find $X$ as the spectrum of $\mathcal{A}$, i.e., the space of maximal ideals. $X \mapsto C(X)$ and the Gelfand functor $\mathcal{A} \mapsto \operatorname{spec} \mathcal{A}$ are in fact mutually inverse category equivalences. Thus, from this perspective the space $X$ and the coordinate ring (ring of "position operators") contain the same information.

It was Connes' fantastic discovery that this correspondence between space and algebra can be pushed much further in the differentiable category. The basic object is now a (locally convex) algebra $\mathscr{A}$ (playing the role of $C^{\infty}(M)$ ). Indispensable basic tools on differentiable manifolds are differential forms and de Rham cohomology. The natural replacement for differential forms is the Hochschild cohomology $H^{n}(\mathscr{A})$ which is the cohomology of the complex $\left(C^{n}(\mathscr{A}), b\right)$ where $C^{n}(\mathscr{A})$ are the (continuous) linear functionals on the $(n+1)$-fold tensor product $\mathscr{A} \otimes \cdots \otimes \mathscr{A}$ and $b: C^{n}(\mathscr{A}) \rightarrow C^{n+1}(\mathscr{A})$ is the well-known Hochschild boundary map

$$
b \phi\left(a_{0}, \ldots, a_{n+1}\right)=\sum_{j=0}^{n}(-1)^{j} \phi\left(a_{0}, \ldots, a_{j} a_{j+1}, \ldots, a_{n+1}\right)+(-1)^{n+1} \phi\left(a_{n+1} a_{0}, a_{1}, \ldots, a_{n}\right) .
$$


Restricting $b$ to the cyclic cochains $C_{\lambda}^{n}(\mathscr{A})^{5}$ yields another complex whose cohomology is the cyclic cohomology $H C^{n}(\mathscr{A})$. The celebrated Hochschild-Kostant-Rosenberg-Connes Theorem says that for a compact smooth manifold the map which sends a de Rham current $C$ (i.e., an element in the dual space of differential forms) to the linear form

$$
\varphi_{C}\left(f_{0}, \ldots, f_{n}\right):=\left\langle C, f_{0} d f_{1} \wedge \cdots \wedge d f_{n}\right\rangle
$$

induces firstly an isomorphism between de Rham currents and the Hochschild cohomology $H^{\bullet}\left(C^{\infty}(M)\right)$ and secondly an isomorphism between $\left(\mathbb{Z}_{2}\right.$-graded) de Rham homology and the (periodic) cyclic cohomology of the algebra $C^{\infty}(M)$. Under this correspondence the exterior derivative (on currents) is sent to (a multiple of) Connes' operator $B: C^{n}(\mathscr{A}) \rightarrow C^{n-1}(\mathscr{A})$,

$$
\begin{aligned}
B \phi\left(a_{0}, \ldots, a_{n-1}\right)= & \sum_{j=0}^{n-1}(-1)^{(n-1) j} \phi\left(1, a_{j}, \ldots, a_{n-1}, a_{0}, \ldots, a_{n-1}\right) \\
& -\sum_{j=0}^{n-1}(-1)^{(n-1) j} \phi\left(a_{j}, 1, a_{j+1}, \ldots, a_{n}, a_{0}, \ldots, a_{j-1}\right) .
\end{aligned}
$$

$B$ is important for a double complex realization of cyclic cohomology. In fact $B b=-b B, B^{2}=0$, $b^{2}=0$ and hence one obtains a double complex with entries $C^{n, m}(\mathscr{A}):=C^{n-m}(\mathscr{A})$ and the total cohomology of this double complex is the periodic cyclic cohomology.

The story is getting even more interesting when it comes to index theory. Given a closed (even-dimensional) spin manifold with its Dirac operator $D$, the Dirac operator determines a spectral triple $\left(\mathscr{A}=C^{\infty}(M), L^{2}(S), D\right)$ where $L^{2}(S)$ is the Hilbert space of $L^{2}$-sections of the spinor bundle (the space of spinor fields). The algebra $\mathscr{A}$ obviously acts by multiplication as bounded operators on $L^{2}(S)$, and we have that $[D, a]$ is bounded for all $a \in \mathscr{A}$.

It should be noted first that the spectral triple $\left(\mathscr{A}, L^{2}(S), D\right)$ is as good as the Riemannian manifold $(M, g)$ : first the geodesic distance can easily be reconstructed from the spectral triple, i.e. $[24$, p. 544$]$

$$
d(p, q)=\sup \{|a(p)-a(q)| \mid a \in \mathscr{A},\|[D, a]\| \leq 1\} .
$$

Even more, Connes' Spin Manifold Theorem [54, Chapter 11] in particular implies that the Riemannian metric may be recovered from the spectral triple. If one assumes more structure on the spectral triple (axioms of a noncommutative spin geometry) then even the manifold may be reconstructed from a noncommutative spin geometry with commutative algebra $\mathscr{A}$. The list of axioms is a bit lengthy, though. The first attempt [85] to prove that Connes' original list of axioms suffices fell short of its goal as was observed by the reviewer of [85]. Quite recently, the result was proved for a slightly stronger set of axioms [86].

An abstract spectral triple $(\mathscr{A}, H, D)^{6}$ is therefore the natural noncommutative model of a smooth Riemannian manifold. Spectral triples come in two flavors, even and odd. Not surprisingly, the spectral triple of a spin manifold is even/odd if the dimension of the manifold is even/odd. To explain the basic index theory in the noncommutative world let us restrict ourselves to the even case. An even spectral triple comes with a chirality operator $\gamma$ which anticommutes with $D$. For any idempotent $e$ (vector bundle, projective module) in the matrix algebra $M_{k}(\mathscr{A})$ we then obtain a Fredholm operator $e D^{+} e: e\left(H^{+}\right)^{k} \longrightarrow e\left(H^{-}\right)^{k}$. Its index is given by the pairing between the K-homology class of $(\mathscr{A}, H, D)$ and the K-theory class of $e$. Taking Chern characters (in (entire) periodic cyclic (co)homology) we have

$\operatorname{ind}\left(e D^{+} e\right)=\left\langle\mathrm{Ch}^{\bullet}(D), \operatorname{ch}_{\bullet}(e)\right\rangle$.

\footnotetext{
${ }^{5} \varphi\left(a_{n}, a_{0}, \ldots, a_{n-1}\right)=(-1)^{n} \varphi\left(a_{0}, \ldots, a_{n}\right)$

${ }^{6} \mathscr{A}$ an algebra represented as bounded operators on the Hilbert space $H, D$ an unbounded self-adjoint operator in $H,[D, a]$ bounded for $a \in \mathscr{A}$.
} 
Here,

$$
\operatorname{ch}(e):=\operatorname{tr}_{0}(e)+\sum_{j=1}^{\infty}(-1)^{j} \frac{(2 j) !}{j !} \operatorname{tr}_{2 j}\left(\left(e-\frac{1}{2}\right) \otimes e^{\otimes(2 j)}\right),
$$

is the Chern character in K-theory ${ }^{7}$. $\mathrm{Ch}^{\bullet}(D)$ is the JLO-Chern character, i.e.,

$$
\mathrm{Ch}^{n}(D)\left(a_{0}, \ldots, a_{k}\right)=\int_{\Delta_{n}} \operatorname{tr}\left(\gamma a_{0} e^{-\sigma_{0} D^{2}}\left[D, a_{1}\right] e^{-\sigma_{1} D^{2}} \cdots\left[D, a_{n}\right] e^{-\sigma_{n} D^{2}}\right) d \sigma
$$

where $\Delta_{n}:=\left\{\left(\sigma_{0}, \ldots, \sigma_{n}\right) \in \mathbb{R}^{n+1} \mid \sigma_{j} \geq 0, \sigma_{0}+\cdots+\sigma_{n}=1\right\}$ is the standard $n$-simplex.

Replacing $D$ by $\sqrt{t} D, t>0$, does not change the left and right hand sides of (4.12). Under the assumption that we do have short-time asymptotic expansions like (4.10) in our spectral triple ${ }^{8}$ it was shown in [25] that also the JLO-cocycle $\mathrm{Ch}^{n}(\sqrt{t} D)$ has short time asymptotic expansions.

The transgression formula

$$
-\frac{d}{d t} \mathrm{Ch}^{n}(t D)=b \phi \mathrm{h}^{n-1}(t D, D)+B \phi \mathrm{h}^{n+1}(t D, D)
$$

(the expression for $\phi \mathrm{h}$ is explicitly known) then shows that the JLO-cocycle may be replaced by a cohomologous cocycle consisting of "local data" which still computes the index (4.12). The details of this are the content of the celebrated Local Index Theorem in noncommutative geometry of Connes and Moscovici [25].

\section{Noncommutative geometry and the standard model with gravity}

The full action of the standard model of particle physics is quite involved and spelled out it fills about a page [23, Section 4.1]. It has been known for a long time that bits of the standard model Lagrangian are intimately related to the heat trace expansion (4.10). To be more specific, let $D$ be a Dirac type operator on a closed manifold. Recall that locally a Dirac operator has the form $g^{i j} c\left(\partial_{i}\right) \partial_{j}+\cdots$, i.e. its leading symbol at a (co)tangent vector $v$ is given by Clifford multiplication by $v$. Consequently its square is an operator of Laplace type which locally takes the form $D^{2}=g^{i j} \partial_{i} \partial_{j}+\cdots$.

The heat trace expansion (4.10) takes the form

$$
\operatorname{Tr}\left(e^{-t D^{2}}\right) \sim_{t \rightarrow 0+} \sum_{j=0}^{\infty} A_{j / 2}\left(D^{2}\right) t^{(j-\operatorname{dim} M) / 2},
$$

where $A_{j / 2}\left(D^{2}\right)=\int_{M} a_{j / 2}\left(x ; D^{2}\right) d \operatorname{vol}_{M}(x)$ is the integral of a local expression $a_{j / 2}\left(x ; D^{2}\right)$ in the metric, the fibre metric, and the coefficients of $D$. From the very construction it is immediately clear that the functional $A_{j / 2}\left(D^{2}\right)$ is invariant under the natural action of the diffeomorphism group. Such functionals are not easy to find from scratch and the complexity of the expression for $A_{j / 2}$ increases fast. For differential operators $A_{j / 2}=0$ for $j$ odd. For a Dirac operator on a Clifford bundle $E$, the first few $A_{j}^{\prime} s$ are known explicitly. We mention

$$
a_{0}\left(x, D^{2}\right)=(4 \pi)^{-\operatorname{dim} M / 2} \operatorname{rank}(E), \quad A_{0}\left(D^{2}\right)=(4 \pi)^{-\operatorname{dim} M / 2} \operatorname{rank}(E) \operatorname{vol}(M),
$$

\footnotetext{
${ }^{7} \operatorname{tr}_{0}: M_{n}(\mathscr{A}) \rightarrow A$ is the generalized trace map, $\operatorname{tr}_{2 j}$ is its obvious extension to tensor products.

${ }^{8}$ The proper notion is that of a spectral triple with discrete dimension spectrum.
} 


$$
a_{1}\left(x, D^{2}\right)=(4 \pi)^{-\operatorname{dim} M / 2} \operatorname{tr}\left(-\frac{R}{6}+\mathcal{E}\right),
$$

where $R$ denotes the scalar curvature and $\mathcal{E}=\nabla^{*} \nabla-D^{2}$ is the 0th order term in the Lichnerowicz formula for $D$. Hence, for the spin Dirac operator $A_{1}\left(D^{2}\right)$ is nothing but the Einstein action.

The appearance of the Einstein action in the heat trace expansion may lead to the following speculation: can one cook up a Dirac operator in such a way that the full action of the standard model appears in the short-time (i.e. high energy expansion) of the heat trace? The answer to this question is probably no. However, the stunning result of Chamseddine, Connes and Marcolli [23] is that the answer is yes if we consider the Dirac operator on a mildly noncommutative space.

To explain this, we first summarize a few relations between the heat trace, the $\zeta$-function and the non-commutative residue. Again, let $D$ be a Dirac type operator on the closed manifold $M$. For a pseudodifferential operator $A$ the noncommutative residue is defined by

$$
\begin{aligned}
f A & :=\operatorname{Res}_{s=0} \operatorname{Tr}\left(A|D|^{-s}\right) \\
& =-2 \text { times the coefficient of } \log t \text { in the asymptotic expansion of } \operatorname{Tr}\left(A e^{-t D^{2}}\right) .
\end{aligned}
$$

The noncommutative residue is indeed independent of the choice of $D$. For the Dirac operator itself there is an obvious relation between the noncommutative residue of $|D|^{-k}$ and the heat coefficients. Namely, for a non-negative integer $k$

$$
f|D|^{-k}=\operatorname{Res}_{s=k} \zeta_{|D|}(s)=\frac{2 A_{(\operatorname{dim} M-k) / 2}\left(D^{2}\right)}{\Gamma(k / 2)}
$$

(with the understanding $1 / \Gamma(0)=0$ ).

Using simple properties of the Mellin transform, one therefore derives the following result (cf. [23, Appendix]): if $f$ is an even smooth rapidly decaying function on the real line then one has the following asymptotic expansion:

$$
\begin{aligned}
\operatorname{Tr}(f(D / \Lambda)) & \sim_{\Lambda \rightarrow \infty} f(0) A_{\frac{1}{2} \operatorname{dim} M}\left(D^{2}\right) \Lambda^{0}+\sum_{k=1}^{\operatorname{dim} M} f_{k} f|D|^{-k} \Lambda^{k}+o(1) \\
& \sim_{\Lambda \rightarrow \infty} f(0) A_{\frac{1}{2} \operatorname{dim} M}\left(D^{2}\right) \Lambda^{0}+\sum_{k=1}^{\operatorname{dim} M} f_{k} \frac{2 A_{(\operatorname{dim} M-k) / 2}\left(D^{2}\right)}{\Gamma(k / 2)} \Lambda^{k}+o(1),
\end{aligned}
$$

where $f_{k}=\int_{0}^{\infty} u^{k-1} f(u) d u$. This asymptotic expansion is the key for the understanding of the high energy expansion of the spectral action.

Some decade ago A. Chamseddine and A. Connes [22] proposed the spectral action

$$
\operatorname{Tr}\left(f(D / \Lambda)+\frac{1}{2}\langle J \psi, D \psi\rangle\right.
$$

on a noncommutative space $(\mathscr{A}, \mathscr{H}, D)$. The stunning relation to the standard model is as follows.

Let $M$ be a closed 4-dimensional spin manifold. Furthermore, let $F$ be a finite spectral triple over the algebra $\mathscr{A}=\mathbb{C} \oplus \mathbb{H} \oplus M_{3}(\mathbb{C})$. The algebra $\mathscr{A}$ is deliberately chosen such that the natural group of local gauge transformations of the space $M \times F$ is the semidirect product of $C^{\infty}(M, \mathrm{U}(1) \times \mathrm{SU}(2) \times \mathrm{SU}(3))$ by the diffeomorphism group of $M$.

The spin Dirac operator on $M$ and the Dirac operator on the finite spectral triple $(\mathscr{A}, H, D)$ (this is after all a self-adjoint endomorphism of a finite-dimensional Hilbert space) give rise to 
a spectral triple $\left(C^{\infty}(M, \mathscr{A}), \mathscr{H}, D\right)$ over the noncommutative algebra $C^{\infty}(M, \mathscr{A})$. The Dirac operator on the finite spectral triple $(\mathscr{A}, H, D)$ depends on 31 real parameters, which physically correspond to the masses for leptons and quarks etc. The main result of [23] states that the asymptotic formula $\Lambda \rightarrow \infty$ for the spectral action functional (5.2) yields the full Lagrangian of the standard model with neutrino mixing and Majorana mass terms. Note that through the spin manifold $M$ gravity is naturally built into the model.

Let us look at (5.1). Then the result of [23] says that for the noncommutative spectral triple sketched above the Lagrangian of the standard model shows up in the coefficients of the expansion (5.1). This is a far reaching generalization of the above observation that the Einstein action is the $A_{1}$-term in the expansion for the spin Dirac operator.

\section{Heat-kernel asymptotics and quantum gravity}

In classical and quantum field theory, as well as in the current attempts to develop a quantum theory of the universe and of gravitational interactions, it remains very useful to describe physical phenomena in terms of differential equations for the variables of the theory, supplemented by boundary conditions for the solutions of such equations. For example, the problems of electrostatics, the analysis of waveguides, the theory of vibrating membranes, the Casimir effect, van der Waals forces, and the problem of how the universe could evolve from an initial state, all need a careful assignment of boundary conditions. In the latter case, if one follows a functional-integral approach, one faces two formidable tasks: (i) the specification of the geometries occurring in the "sum over histories" and matching the assigned boundary data; (ii) the choice of boundary conditions on metric perturbations which may lead to the evaluation of the one-loop semiclassical approximation.

Indeed, while the full functional integral for quantum gravity is a fascinating idea but remains a formal tool, the one-loop calculation may be put on solid ground, and appears particularly interesting because it yields the first quantum corrections to the underlying classical theory (although it is well known that quantum gravity based on Einstein's theory is not perturbatively renormalizable). Within this framework, it is of crucial importance to evaluate the one-loop divergences of the theory under consideration. Moreover, the task of the theoretical physicist is to understand the deeper general structure of such divergences. For this purpose, one has to pay attention to all geometric invariants of the problem, in a way made clear by a branch of mathematics known as invariance theory. The key geometric elements of our problem are hence as follows.

A Riemannian geometry $(M, g)$ is given, where the manifold $M$ is compact and has a boundary $\partial M$ with induced metric $\gamma$, and the metrics $g$ and $\gamma$ are positive-definite. A vector bundle over $M$, say $V$, is given, and one has also to consider a vector bundle $\widetilde{V}$ over $\partial M$. An operator of Laplace type, say $P$, is a second-order elliptic operator with leading symbol given by the metric (more precisely, it has scalar leading symbol $g^{a b} k_{a} k_{b}$ ). Thus, one deals with a map from the space of smooth sections of $V$ onto itself,

$$
P: C^{\infty}(M, V) \rightarrow C^{\infty}(M, V),
$$

which can be expressed in the form

$$
P=-g^{a b} \nabla_{a}^{V} \nabla_{b}^{V}-E
$$

where $\nabla^{V}$ is the connection on $V$, and $E$ is an endomorphism of $V: E \in \operatorname{End}(V)$. Moreover, the boundary operator is a map

$$
\mathcal{B}: C^{\infty}(M, V) \rightarrow C^{\infty}(\partial M, \widetilde{V})
$$


and contains all the informations on the boundary conditions of the problem. Since gauge theories need a generalization of Robin boundary conditions, we consider a boundary operator of the form (the operation of restriction to the boundary being implicitly understood)

$$
\mathcal{B}=\nabla_{N}+\frac{1}{2}\left[\Gamma^{i} \widehat{\nabla}_{i}+\widehat{\nabla}_{i} \Gamma^{i}\right]+S
$$

With our notation, $\nabla_{N}$ is the normal derivative operator $\nabla_{N} \equiv N^{a} \nabla_{a}$ ( $N^{a}$ being the inwardpointing normal to $\partial M), S$ is an endomorphism of the vector bundle $\widetilde{V}, \Gamma^{i}$ are endomorphismvalued vector fields on $\partial M$, and $\widehat{\nabla}_{i}$ denotes tangential covariant differentiation with respect to the connection induced on $\partial M$. More precisely, when sections of bundles built from $V$ are involved, $\widehat{\nabla}_{i}$ means

$$
\nabla_{\partial M}^{(\mathrm{lc})} \otimes 1+1 \otimes \nabla
$$

where $\nabla_{\partial M}^{(\mathrm{lc})}$ denotes the Levi-Civita connection of the boundary of $M$. Hereafter, we assume that $1+\Gamma^{2}>0$, to ensure strong ellipticity of the boundary value problem (see definition on pp. 69-70 of [44]).

The case of mixed boundary conditions corresponds to the possibility of splitting the bundle $V$, in a neighbourhood of $\partial M$, as the direct sum of two bundles, say $V_{1}$ and $V_{2}$, for each of which a boundary operator of the Dirichlet or (generalized) Robin type is also given. The former involves a projection operator, say $\Pi$, while the latter may also involve the complementary projector, $1-\Pi$, and the metric of $V$, say $H$ :

$$
\mathcal{B}_{1}=\Pi, \quad \mathcal{B}_{2}=(1-\Pi)\left[H \nabla_{N}+\frac{1}{2}\left(\Gamma^{i} \widehat{\nabla}_{i}+\widehat{\nabla}_{i} \Gamma^{i}\right)+S\right]
$$

We can now come back to our original problem, where only the boundary operator (6.1) occurs, and investigate its effect on heat-kernel asymptotics. Indeed, given the heat equation for the operator $P$, its kernel, i.e. the heat kernel, is, by definition, a solution for $t>0$ of the equation

$$
\left(\frac{\partial}{\partial t}+P\right) U\left(x, x^{\prime} ; t\right)=0
$$

jointly with the initial condition

$$
\lim _{t \rightarrow 0} \int_{M} d x^{\prime} \sqrt{\operatorname{det} g\left(x^{\prime}\right)} U\left(x, x^{\prime} ; t\right) \rho\left(x^{\prime}\right)=\rho(x),
$$

and the boundary condition

$$
\left[\mathcal{B} U\left(x, x^{\prime} ; t\right)\right]_{\partial M}=0 \text {. }
$$

The fibre trace of the heat-kernel diagonal, i.e. $\operatorname{Tr} U(x, x ; t)$, admits an asymptotic expansion which describes the local asymptotics, and involves interior invariants and boundary invariants. Interior invariants are built universally and polynomially from the metric, the Riemann curvature $R_{b c d}^{a}$ of $M$, the bundle curvature, say $\Omega_{a b}$, the endomorphism $E$, and their covariant derivatives. By virtue of Weyl's work on the invariants of the orthogonal group, these polynomials can be found by using only tensor products and contraction of tensor arguments. The asymptotic expansion of the integral

$$
\int_{M} d x \sqrt{\operatorname{det} g} \operatorname{Tr} U(x, x ; t) \equiv \operatorname{Tr}_{L^{2}}\left(e^{-t P}\right),
$$


yields instead the global asymptotics. For our purposes, it is more convenient to weight $e^{-t P}$ with a smooth function $f \in C^{\infty}(M)$, and then consider the asymptotic expansion

$$
\operatorname{Tr}_{L^{2}}\left(f e^{-t P}\right) \equiv \int_{M} d x \sqrt{\operatorname{det} g} f(x) \operatorname{Tr} U(x, x ; t) \sim(4 \pi t)^{-m / 2} \sum_{l=0}^{\infty} t^{l / 2} A_{l / 2}(f, P, \mathcal{B}) .
$$

Hereafter, $m$ is the dimension of $M$, and the coefficient $A_{l / 2}(f, P, \mathcal{B})$ consists of an interior part, say $C_{l / 2}(f, P)$, and a boundary part, say $B_{l / 2}(f, P, \mathcal{B})$. The interior part vanishes for all odd values of $l$, whereas the boundary part only vanishes if $l=0$. The interior part is obtained by integrating over $M$ the linear combination of local invariants of the appropriate dimension mentioned above, where the coefficients of the linear combination are universal constants, independent of $m$. Moreover, the boundary part is obtained upon integration over $\partial M$ of another linear combination of local invariants. In that case, however, the structure group is $O(m-1)$, and the coefficients of linear combination are universal functions, independent of $m$, unaffected by conformal rescalings of $g$, and invariant in form (i.e. they are functions of position on the boundary, whose form is independent of the boundary being curved or totally geodesic). It is thus clear that the general form of the $A_{l / 2}$ coefficient is a well posed problem in invariance theory, where one has to take all possible local invariants built from $f, R_{b c d}^{a}, \Omega_{a b}, K_{i j}, E, S, \Gamma^{i}$ and their covariant derivatives (hereafter, $K_{i j}$ is the extrinsic-curvature tensor of the boundary), eventually integrating their linear combinations over $M$ and $\partial M$. For example, in the boundary part $B_{l / 2}(f, P, \mathcal{B})$, the local invariants integrated over $\partial M$ are of dimension $l-1$ in tensors of the same dimension of the second fundamental form of the boundary, for all $l \geq 1$. The universal functions associated to all such invariants can be found by using the conformal-variation method described, for example, in [44], jointly with the analysis of simple examples and particular cases.

In other words, recurrence relations of algebraic nature exist among all universal functions, and one can therefore use the solutions of simple problems to determine completely the remaining set of universal functions for a given value of the integer $l$ in the asymptotic expansion (6.2). The detailed investigation of the coefficients $A_{1}, A_{3 / 2}$ and $A_{2}$ when the boundary operator is given by equation (6.1) and all curvature terms are non-vanishing is performed in [4, 38] One then finds the result (which holds for all integer values of $l \geq 2$ )

$$
A_{l / 2}(f, P, \mathcal{B})=\widetilde{A}_{l / 2}(f, P, \mathcal{B})+\int_{\partial M} \operatorname{Tr}\left[a_{l / 2}(f, R, \Omega, K, E, \Gamma, S)\right],
$$

where $\widetilde{A}_{l / 2}(f, P, \mathcal{B})$ is formally analogous to the purely Robin case, but replacing the universal constants in the boundary terms with universal functions, whereas $a_{l / 2}$ is a linear combination of all local invariants of the given dimension which involve contractions with $\Gamma^{i}$. Our task is now to derive an algorithm for the general form of $a_{l / 2}$, since it helps a lot to have a formula that clarifies the general features of a scheme where the number of new invariants is rapidly growing. Indeed, from [4,38], we know that, in $a_{1}$, only one new invariant occurs: $f K_{i j} \Gamma^{i} \Gamma^{j}$, whereas in $a_{3 / 2} 11$ new invariants occur, obtained by contraction of $\Gamma^{i}$ with terms like (tensor indices are here omitted for simplicity)

$$
f K^{2}, f K S, f \widehat{\nabla} K, f \widehat{\nabla} S, f R, f \Omega, f_{; N} K .
$$

In $a_{2}$, the number of new invariants is 68: 57 involve contractions of $\Gamma^{i}$ with terms like

$$
\begin{aligned}
& f K^{3}, f K^{2} S, f K S^{2}, f R K, f \Omega K, f E K, f R S, f \Omega S, f K \widehat{\nabla} K, f S \widehat{\nabla} K, \\
& f K \widehat{\nabla} S, f S \widehat{\nabla} S, f \widehat{\nabla} \widehat{\nabla} K, f \widehat{\nabla} \widehat{\nabla} S, f \nabla R, f \nabla \Omega, f \nabla E,
\end{aligned}
$$

10 local invariants involve contractions of $\Gamma^{i}$ with contributions like

$$
f_{; N} K^{2}, f_{; N} K S, f_{; N} \widehat{\nabla} K, f_{; N} \widehat{\nabla} S, f_{; N} R, f_{; N} \Omega,
$$


and the last invariant is $f_{; N N} K_{i j} \Gamma^{i} \Gamma^{j}$. It is thus clear that the knowledge of all local invariants in $a_{l / 2}$ plays a role in the form of $a_{(l+1) / 2}$, and one can write the formulas

$$
\begin{aligned}
& a_{1}=f \sum_{i=1}^{i_{1}} \mathcal{U}_{i}^{(1,1)} I_{i}^{(1)} \\
& a_{3 / 2}=f \sum_{i=1}^{i_{2}} \mathcal{U}_{i}^{(3 / 2,3 / 2)} I_{i}^{(3 / 2)}+f_{; N} \sum_{i=1}^{i_{1}} \mathcal{U}_{i}^{(3 / 2,1)} I_{i}^{(1)} \\
& a_{2}=f \sum_{i=1}^{i_{3}} \mathcal{U}_{i}^{(2,2)} I_{i}^{(2)}+f_{; N} \sum_{i=1}^{i_{2}} \mathcal{U}_{i}^{(2,3 / 2)} I_{i}^{(3 / 2)}+f_{; N N} \sum_{i=1}^{i_{1}} \mathcal{U}_{i}^{(2,1)} I_{i}^{(1)} .
\end{aligned}
$$

With our notation, $i_{1}=1, i_{2}=10, i_{3}=57$, and $\mathcal{U}_{i}^{(x, y)}$ are the universal functions, where $i$ is an integer $\geq 1, x$ is always equal to the order $l / 2$ of $a_{l / 2}$, and $y$ is equal to the label of the invariant $I_{i}^{(y)}$, which does not contain $f$ or derivatives of $f$ and is of dimension $2 y-1$ in $K$ or in tensors of the same dimension of $K$.

These remarks make it possible to write down a formula which holds for all $l \geq 2$ :

$$
a_{l / 2}(f, R, \Omega, K, E, \Gamma, S)=\sum_{r=0}^{l-2} f^{(r)} \sum_{i=1}^{i_{l-r-1}} \mathcal{U}_{i}^{(l / 2,(l-r) / 2)}\left[\Gamma^{2}\right] I_{i}^{(l-r) / 2}[R, \Omega, K, E, \Gamma, S],
$$

where $f^{(r)}$ is the normal derivative of $f$ of order $r$ (with $f^{(0)}=f$ ), and square brackets are used for the arguments of universal functions and local invariants, respectively. The equations (6.3) and (6.4) represent the desired parametrization of heat-kernel coefficients with generalized boundary conditions, provided that the $\Gamma^{i}$ are covariantly constant.

One has now to evaluate the universal functions in the general formulas for $A_{3 / 2}, A_{2}, A_{5 / 2}$ and so on. For the coefficients $A_{3 / 2}$ and $A_{2}$, results of a limited nature are available in [4, 38], which show that all universal functions are generated from $\sqrt{1+\Gamma^{2}}$ and $\frac{1}{\sqrt{-\Gamma^{2}}} \operatorname{Artanh} \sqrt{-\Gamma^{2}}$. Upon completion of this hard piece of work, one could perform the evaluation of all universal functions for $A_{5 / 2}(f, P, \mathcal{B})$ as well, possibly with the help of computers. For this purpose, one has to combine the conformal-variation method with the analysis of simpler cases. One then obtains a quicker and more elegant derivation of the coefficient $A_{1}(f, P, \mathcal{B})$. There are thus reasons to expect that, in the near future, all heat-kernel coefficients with generalized boundary conditions may be obtained via a computer algorithm in a relatively short time. This adds evidence in favour of the understanding of general mathematical structures being very helpful in providing the complete solution of difficult problems in physics and mathematics. In particular, from the point of view of quantum field theory in curved manifolds, this would mean an entirely geometric understanding of the first set of quantum corrections to the underlying classical theory, with the help of invariance theory, functorial methods and computer programs. This is the case because, on the one hand, heat-kernel coefficients $A_{l / 2}(f, P, \mathcal{B})$ in the asymptotic expansion (6.2) are obtained from a geometric construction as we said, while, on the other hand, the coefficient $A_{l / 2}$ yields the one-loop divergence of the corresponding theory in $l$-dimensional space-time. Such one-loop divergences are indeed the first set of quantum corrections to the classical theory. This has implications for the old unification program of Section 2, because it shows the perturbative limits of its quantization: one-loop quantum gravity based on the Einstein-Hilbert action is only on-shell finite, i.e. upon imposing the vacuum Einstein equations

$$
R_{a b}-\frac{1}{2} g_{a b} R=0 \quad \Longrightarrow \quad R_{a b}=0 .
$$

The reason is that only three geometric invariants can be built in such a case, i.e.

$$
R_{a b c d} R^{a b c d}, \quad R_{a b} R^{a b}, \quad R^{2},
$$


bearing in mind that the fourth one, given by the wave operator acting on the scalar curvature, has vanishing integral if spacetime has empty boundary. The above invariants should be integrated over the spacetime manifold, so that the integral representation of the Euler number makes it possible to deal eventually with two invariants only: $R_{a b} R^{a b}$ and $R^{2}$. But both of them vanish if and only if the metric solves the vacuum Einstein equations in four dimensions.

In Euclidean quantum gravity, if one uses the de Donder gauge-fixing functional, one finds a boundary operator formally analogous to the one in equation (6.1) but imcompatible with the strong ellipticity of the boundary value problem [5]. Only recently, in [45, 46], has one found a viable way out in the particular case of the Euclidean 4-ball, and more work is in order on this key issue, which has also implications for singularity avoidance in quantum cosmology, as we mentioned in Section 3. This framework is relevant also for the new unification outlined in Section 2, as is shown in [8]. In this work, the authors study a toy model of brane-induced gravity for the calculation of its one-loop effective action, and obtain the inverse-mass asymptotic expansion of the effective action and its ultraviolet divergences, which are found to be nonvanishing in all spacetime dimensions. They also obtain the heat-kernel asymptotics associated with generalized boundary conditions containing tangential derivatives (cf. equation (6.1)). In addition to the usual powers of the $t$-parameter in the expansion (6.2), they find also logarithmic terms or powers multiple of one quarter. Such a property is considered in the context of strong ellipticity of the boundary value problem, which can be violated under certain conditions [8].

\section{A Infinite-dimensional manifolds in quantum gravity}

We would like to stress here that infinite-dimensional manifolds are the natural arena for studying the quantization of the gravitational field, even prior to considering a space-of-histories formulation. In [51], an approach is proposed where the states and operators emerge as certain scalar and vector fields on an infinite-dimensional manifold $\mathcal{G}$ of classical solutions of Einstein's equations. Such a formalism essentially reproduces quantum electrodynamics when $\mathcal{G}$ is replaced by the space of square-integrable solutions of Maxwell's equations. According to the author of [51], a quantum theory of gravitation is obtained if one can solve two problems:

1. Impose a manifold structure on a suitable collection of solutions of the Einstein equations (e.g. without sources).

2. Determine tensor fields $T_{a}^{b}$ and $G^{a b}$ on that manifold, subject to

$$
T_{a}^{b} T_{b}^{c}=-\delta_{a}^{c}, \quad G^{a b}=G^{b a}, \quad T_{c}^{a} G^{c b}=-T_{c}^{b} G^{c a} .
$$

The solution of these two problems for any classical system of interacting bosonic fields should produce a quantum theory. In general relativity, one might select for $\mathcal{G}$ the collection of all asymptotically simple solutions of the Einstein equations, the main steps being as follows [51]. Let $\bar{M}=M \cup \mathcal{I}$ be a manifold with boundary, where the boundary $\mathcal{I}$ consists of two disjoint copies of $S^{2} \times \mathbb{R}$, and the interior of $M$ is $\mathbb{R}^{4}$. The main idea is to incorporate into $\bar{M}$ all structure which is independent of the metric chosen. One therefore requires that a conformal factor $\Omega$ is given on $\bar{M}$ such that

$$
\Omega>0 \text { on } M, \quad \Omega=0 \text { on } \mathcal{I},
$$

while the gradient of $\Omega$ is nonvanishing on $\mathcal{I}$, whose conformal structure is required to be given. Let now $\mathcal{G}$ be the collection of all source-free solutions of the Einstein equations on $M$ which are asymptotically simple with conformal factor $\Omega$ and conformal infinity $\mathcal{I}$, and which reproduce the given conformal structure on $\mathcal{I}$. A vector in $\mathcal{G}$ at a point $g \in \mathcal{G}$ (the point $g$ being a solution of the Einstein equations) is a linearized solution $\gamma_{\alpha}^{\beta}$ which is regular on $\mathcal{I}$. Thus, the desired 
tensor field $T_{a}^{b}$ is a linear mapping on linearized solutions at $g$ which, applied twice to $\gamma_{\alpha}^{\beta}$, gives $-\gamma_{\alpha}^{\beta}$. One could obtain such a mapping by expressing the linearized solution in terms of its initial data on past null infinity, and then altering the phase of the data in a suitable way. Last, but not least, the desired metric $G_{a b}$ on $\mathcal{G}$ should assign a real number to each linearized solution. One might use for this purpose the integral [51]

$$
I \equiv \int_{M} \gamma_{\alpha}^{\beta} \gamma_{\beta}^{\alpha} d V
$$

To sum up, there are at least three sources of infinite-dimensionality in quantum gravity:

1. The infinite-dimensional Lie group (or pseudo-group) of spacetime diffeomorphisms, which is the invariance group of general relativity in the first place [28, 93].

2. The infinite-dimensional space of histories in a functional-integral quantization [30, 31].

3. The infinite-dimensional Geroch space of asymptotically simple spacetimes [51].

\section{Acknowledgments}

G. Esposito is grateful to the INFN for financial support, and to IMFUFA (Roskilde University), where part of this material was first presented.

This paper is based on joint discussions among the authors when preparing the Summer School 2008 on "Quantum Gravity", see http://QuantumGravity.ruc.dk.

\section{References}

[1] Angelantonj C., Sagnotti A., Open strings, Phys. Rep. 371 (2002), 1-150, hep-th/0204089.

[2] Atiyah M.F., Harmonic spinors and elliptic operators. Arbeitstagung Lecture, 16 July 1962, Notes by S. Lang, duplicated, Bonn, 1962.

[3] Atiyah M.F., Bott R., Patodi V.K., On the heat equation and the index theorem, Invent. Math. 19 (1973), 279-330.

[4] Avramidi I.G., Esposito G., New invariants in the one-loop divergences on manifolds with boundary, Classical Quantum Grav. 15 (1998), 281-297.

[5] Avramidi I.G., Esposito G., Gauge theories on manifolds with boundary, Comm. Math. Phys. 200 (1999), 495-543, hep-th/9710048.

[6] Barvinsky A.O., Quantum effective action in spacetimes with branes and boundaries: diffeomorphism invariance, Phys. Rev. D $\mathbf{7 4}$ (2006), 084033, 18 pages, hep-th/0608004.

[7] Barvinsky A.O., Nesterov D.V., Quantum effective action in spacetimes with branes and boundaries, Phys. Rev. D 73 (2006), 066012, 12 pages, hep-th/0512291.

[8] Barvinsky A.O., Kamenshchik A.Yu., Kiefer C., Nesterov D.V., Effective action and heat kernel in a toy model of brane-induced gravity, Phys. Rev. D 75 (2007), 044010, 14 pages, hep-th/0611326.

[9] Becker K., Becker M., Schwarz J.H., String theory and M-theory: a modern introduction, Cambridge University Press, Cambridge, 2007.

[10] Bohle-Carbonell M., Booß B., Jensen J.H., Innermathematical vs. extramathematical obstructions to model credibility, in Proceedings of the Fourth International Conference "Mathematical Modelling in Science and Technology" (August 1983, Zürich), Editor X. Avula, Pergamon Press, New York, 1984, 62-65.

[11] Bonanno A., Reuter M., Cosmology of the Planck era from a renormalization group for quantum gravity, Phys. Rev. D 65 (2002), 043508, 20 pages, hep-th/0106133.

[12] Booß B., Bleecker D.D., Topology and analysis. The Atiyah-Singer index formula and gauge-theoretic physics, Springer, New York, 1985. See http://imfufa.ruc.dk/ Booss/book/index.htm for new edition, in preparation.

[13] Booß-Bavnbek B., Against ill-founded, irresponsible modelling, in Teaching of Mathematical Modelling and Applications, Editors M. Niss et al., Ellis Horwood, Chichester, 1991, 70-82. 
[14] Booß-Bavnbek B., Bleecker D., Spectral invariants of operators of Dirac type on partitioned manifolds, in Aspects of Boundary Problems in Analysis and Geometry, Editors J. Gil et al. Birkhäuser, Basel, 2004, 1-130, math.AP/0304214.

[15] Booß-Bavnbek B., Phillips J., Lesch M., Unbounded Fredholm operators and spectral flow, Canad. J. Math. 57 (2005), 225-250, math.FA/0108014.

[16] Booß-Bavnbek B., Wojciechowski K.P., Elliptic boundary problems for Dirac operators, Birkhäuser, Boston, 1993.

[17] Branson T.P., Gilkey P., Residues of the eta function for an operator of Dirac type, J. Funct. Anal. 108 (1992), 47-87.

[18] Branson T.P., Ørsted B., Explicit functional determinants in four dimensions, Proc. Amer. Math. Soc. 113 (1991), 671-684.

[19] Brüning J., Lesch M., On the eta-invariant of certain non-local boundary value problems, Duke Math. J. 96 (1999), 425-468, dg-ga/9609001.

[20] Brüning J., Lesch M., On boundary value problems for Dirac type operators. I. Regularity and selfadjointness, J. Funct. Anal. 185 (2001), 1-62, math.FA/9905181.

[21] Bytsenko A.A., Cognola G., Elizalde E., Moretti V., Zerbini S., Analytic aspects of quantum fields, World Scientific, Singapore, 2004.

[22] Chamseddine A.H., Connes A., The spectral action principle, Comm. Math. Phys. 186 (1997), 731-750, hep-th/9606001.

[23] Chamseddine A.H., Connes A., Marcolli M., Gravity and the standard model with neutrino mixing, hep-th/0610241.

[24] Connes A., Noncommutative geometry, Academic Press, San Diego, CA, 1994.

[25] Connes A., Moscovici H., The local index formula in noncommutative geometry, Geom. Funct. Anal. 5 (1995), 174-243.

[26] Deligne P., Etingof P., Freed D.S., Jeffrey L.C., Kazhdan P., Morgan J.W., Morrison D.R., Witten E., Quantum fields and strings: a course for mathematicians, American Mathematical Society, Providence, 1999.

[27] Deser S., Zumino B., Consistent supergravity, Phys. Lett. B 62 (1976), 335-337.

[28] DeWitt B.S., Dynamical theory of groups and fields, Gordon \& Breach, New York, 1965.

[29] DeWitt B.S., Quantum theory of gravity. 2. The manifestly covariant theory, Phys. Rev. 162 (1967), 11951239.

[30] DeWitt B.S., The global approach to quantum field theory, Int. Ser. Monogr. Phys., Vol. 114, Clarendon Press, Oxford, 2003.

[31] DeWitt B.S., The space of gauge fields: its structure and geometry, in 50 Years of Yang-Mills Theory, Editor G. 't Hooft, World Scientific, Singapore, 2005, 15-32.

[32] Dirac P.A.M., The fundamental equations of quantum mechanics, Proc. R. Soc. Lond. A 109 (1925), 642653.

[33] Dirac P.A.M., The Lagrangian in quantum mechanics, Phys. Z. USSR 3 (1933), 64-72.

[34] Di Vecchia P., Liccardo A., D-branes in string theory. 1, NATO Adv. Study Inst. Ser. C. Math. Phys. Sci. 556 (2000), 1-59, hep-th/9912161.

[35] Di Vecchia P., Liccardo A., D-branes in string theory. 2, hep-th/9912275.

[36] Donaldson S.K., Kronheimer P.B., The geometry of four-manifolds, The Clarendon Press, Oxford University Press, New York, 1990.

[37] Douglas R.G., Wojciechowski K.P., Adiabatic limits of the $\eta$-invariants. The odd-dimensional AtiyahPatodi-Singer problem, Comm. Math. Phys. 142 (1991), 139-168.

[38] Dowker J.S., Kirsten K., The $a_{3 / 2}$ heat kernel coefficient for oblique boundary conditions, Classical Quantum Gravity 16 (1999), 1917-1936, hep-th/9806168.

[39] Einstein A., The foundation of the general theory of relativity, Annalen Phys. 49 (1916), 769-822.

[40] Ellis G.F.R., Hawking S.W., The cosmic black body radiation and the existence of singularities in our universe, Astrophys. J. 152 (1968), 25-36. 
[41] Elizalde E., On the concept of determinant for the differential operators of quantum physics, JHEP 1999 (1999), no. 07, 015, 13 pages, hep-th/9906229.

[42] Elizalde E., Zeta functions: formulas and applications, J. Comput. ES Appl. Math. 118 (2000), 125-142.

[43] Elizalde E., Vanzo L., Zerbini, S., Zeta function regularization, the multiplicative anomaly and the Wodzicki residue, Comm. Math. Phys. 194 (1998) 613-630, hep-th/9701060.

[44] Esposito G., Dirac operators and spectral geometry, Cambridge Lect. Notes Phys., Vol. 12, Cambridge University Press, Cambridge, 1998.

[45] Esposito G., Fucci G., Kamenshchik A.Yu., Kirsten K., Spectral asymptotics of Euclidean quantum gravity with diff-invariant boundary conditions, Classical Quantum Gravity 22 (2005), 957-974, hep-th/0412269.

[46] Esposito G., Fucci G., Kamenshchik A.Yu., Kirsten K., A non-singular one-loop wave function of the universe from a new eigenvalue asymptotics in quantum gravity, JHEP 2005 (2005), no. 09, 063, 17 pages, hep-th/0507264.

[47] Esposito G., Marmo G., Sudarshan G., From classical to quantum mechanics, Cambridge University Press, Cambridge, 2004.

[48] Faddeev L.D., Popov V.N., Feynman diagrams for the Yang-Mills field, Phys. Lett. B 25 (1967), 29-30.

[49] Feynman R.P., Space-time approach to non-relativistic quantum mechanics, Rev. Mod. Phys. 20 (1948), 367-387.

[50] Geroch R.P., Singularities in closed universes, Phys. Rev. Lett. 17 (1966), 445-447.

[51] Geroch R.P., An approach to quantization of general relativity, Ann. Phys. (N.Y.) 62 (1971), $582-589$.

[52] Gilkey P.B., The residue of the global $\eta$-function at the origin, Adv. Math. 40 (1981), 290-307.

[53] Golfand A.Yu., Likhtman E.P., Extension of the algebra of Poincaré group generators and violation of $p$ invariance, JETP Lett. 13 (1971), 323-326.

[54] Gracia-Bondía J.M., Várilly J.C., Figueroa H., Elements of noncommutative geometry, Birkhäuser, Boston, MA, 2001.

[55] Grubb G., Seeley R.T., Weakly parametric pseudodifferential operators and Atiyah-Patodi-Singer boundary problems, Invent. Math. 121 (1995), 481-529.

[56] Guillemin V., A new proof of Weyl's formula on the asymptotic distribution of eigenvalues, Adv. Math. 55 (1985), 131-160.

[57] Hawking S.W., Occurrence of singularities in open universes, Phys. Rev. Lett. 15 (1965), 689-690.

[58] Hawking S.W., Singularities in the universe, Phys. Rev. Lett. 17 (1966), 444-445.

[59] Hawking S.W., The occurrence of singularities in cosmology, Proc. R. Soc. Lond. A 294 (1966), 511-521.

[60] Hawking S.W., The occurrence of singularities in cosmology. II, Proc. R. Soc. Lond. A 295 (1966), 490-493.

[61] Hawking S.W., The occurrence of singularities in cosmology. III. Causality and singularities, Proc. R. Soc. Lond. A 300 (1967), 187-201.

[62] Hawking S.W., Ellis G.F.R., Singularities in homogeneous world models, Phys. Lett. 17 (1965), $246-247$.

[63] Hawking S.W., Ellis G.F.R., The large-scale structure of space-time, Cambridge University Press, Cambridge, 1973.

[64] Hawking S.W., Penrose R., The singularities of gravitational collapse and cosmology, Proc. R. Soc. Lond. A 314 (1970), 529-548.

[65] Heisenberg W., Quantum-mechanical re-interpretation of kinematic and mechanical relations, Z. Phys. 33 (1925), 879-893.

[66] Horowitz G.T., Marolf D., Quantum probes of spacetime singularities, Phys. Rev. D 52 (1995), 5670-5675, gr-qc/9504028.

[67] Horowitz G.T., Myers R., The value of singularities, Gen. Relativity Gravitation 27 (1995), 915-919, gr-qc/9503062.

[68] Horowitz G.T., Steif A.R., Space-time singularities in string theory, Phys. Rev. Lett. 64 (1990), $260-263$.

[69] Joachim M., Unbounded Fredholm operators and $K$-theory, in High-dimensional Manifold Topology, World Sci. Publ., River Edge, NJ, 2003, 177-199.

[70] Kirk P., Lesch M., The $\eta$-invariant, Maslov index, and spectral flow for Dirac-type operators on manifolds with boundary, Forum Math. 16 (2004), 553-629, math.DG/0012123. 
[71] Lauscher O., Reuter M., Ultraviolet fixed point and generalized flow equation of quantum gravity, Phys. Rev. D 65 (2002), 025013, 44 pages, hep-th/0108040.

[72] Lauscher O., Reuter M., Asymptotic safety in quantum Einstein gravity: nonperturbative renormalizability and fractal spacetime structure, hep-th/0511260.

[73] Lesch M., Determinants of regular singular Sturm-Liouville operators, Math. Nachr. 194 (1998), 139-170, math.DG/9902114.

[74] Lorenz L., On the identity of the vibrations of light with electrical currents, Phil. Mag. 34 (1867), $287-301$.

[75] Lück W., Analytic and topological torsion for manifolds with boundary and symmetry, J. Differential Geom. 37 (1993), 263-322.

[76] Manin Y., Presentation to the closing round table "Are pure and applied mathematics drifting apart?", in Proceedings of the International Congress of Mathematicians (August 22-30, 2006, Madrid), Editors M. Sanz-Solé et al., European Mathematical Society, Zürich, 2007, 762-764, 775-776.

[77] Manin Y., Mathematical knowledge: internal, social and cultural aspects, Mathematics and Culture, Vol. 2, Editors C. Bartocci and P. Odifreddi, 2007, math.HO/0703427.

[78] van Nieuwenhuizen P., Supergravity, Phys. Rep. 68 (1981), 189-398.

[79] Osgood B., Phillips R., Sarnak P., Extremals of determinants of Laplacians, J. Funct. Anal. 80 (1988), $148-211$.

[80] Park J., Wojciechowski K.P., Adiabatic decomposition of the $\zeta$-determinant of the Dirac Laplacian I. The case of invertible tangential operator, Comm. Partial Differential Equations 27 (2002), 1407-1435 (with an Appendix by Y. Lee), math.DG/0112174.

[81] Park J., Wojciechowski K.P., Adiabatic decomposition of the $\zeta$-determinant and scattering theory, Michigan Math. J. 54 (2006), 207-238, math.DG/0111046.

[82] Penrose R., Gravitational collapse and space-time singularities, Phys. Rev. Lett 14 (1965), 57-59.

[83] Polyakov A.M., Quantum geometry of bosonic strings, Phys. Lett. B 103 (1981), 207-210.

[84] Polyakov A.M., Quantum geometry of fermionic strings, Phys. Lett. B 103 (1981), 211-213.

[85] Rennie A., Commutative geometries are spin manifolds, Rev. Math. Phys. 13 (2001), 409-464, math-ph/9903021.

[86] Rennie A., Várilly J.C., Reconstruction of manifolds in noncommutative geometry, math.OA/0610418.

[87] Rarita W., Schwinger J., On a theory of particles with half-integral spin, Phys. Rev. 60 (1941), 61-61.

[88] Reuter M., Nonperturbative evolution equation for quantum gravity, Phys. Rev. D 57 (1998), 971-985, hep-th/9605030.

[89] Reuter M., Saueressig F., Renormalization group flow of quantum gravity in the Einstein-Hilbert truncation, Phys. Rev. D 65 (2002), 065016, 26 pages, hep-th/0110054.

[90] Sakharov A.D., Vacuum quantum fluctuations in curved space and the theory of gravitation, Sov. Phys. Dokl. 12 (1968), 1040-1041.

[91] Scott S.G., Wojciechowski K.P., The $\zeta$-determinant and Quillen determinant for a Dirac operator on a manifold with boundary, Geom. Funct. Anal. 10 (2000), 1202-1236.

[92] Smolin L., The trouble with physics - the rise of string theory, the fall of a science and what comes next, Penguin Books, London, 2006.

[93] Streater R.F., Lost causes in and beyond physics, Springer, New York, 2007.

[94] Vishik S.M., Generalized Ray-Singer conjecture. I. A manifold with a smooth boundary, Comm. Math. Phys. 167 (1995), 1-102, hep-th/9305184.

[95] Volovik G.E., The Universe in a Helium droplet, Int. Ser. Monogr. Phys., Vol. 117, Clarendon Press, Oxford, 2003.

[96] Wess J., Zumino B., Supergauge transformations in four dimensions, Nuclear Phys. B 70 (1974), 39-50.

[97] Wodzicki M., Noncommutative residue. I. Fundamentals, in K-theory, Arithmetic and Geometry (Moscow, 1984-1986), Lecture Notes in Math., Vol. 1289, Springer, Berlin, 1987, 320-399.

[98] Yang C.N., Mills R.L., Conservation of isotopic spin and isotopic gauge invariance, Phys. Rev. 96 (1954), 191-195.

[99] http://aether.lbl.gov/www/projects/cobe; http://map.gsfc.nasa.gov 